\title{
Stability and instability of Ellis and phantom wormholes: Are there ghosts?
}

\author{
K.K. Nandi ${ }^{1,2,3, a}$, A.A. Potapov ${ }^{3, b}$, R.N. Izmailov ${ }^{2, c}$, A.Tamang ${ }^{4, d}$ \\ and \\ J.C. Evans ${ }^{5, e}$ \\ ${ }^{1}$ Department of Mathematics, University of North Bengal, Siliguri 734013, \\ WB, India \\ 2Zel'dovich International Center for Astrophysics, M. Akmullah Bashkir \\ State Pedagogical University, Ufa 450000, RB, Russia \\ ${ }^{3}$ Department of Physics \& Astronomy, Bashkir State University, \\ Sterlitamak Campus, Sterlitamak 453103, RB, Russia \\ ${ }^{4}$ Department of Mathematics, Darjeeling Government College, Richmond \\ Hill, Darjeeling 734104, WB, India \\ ${ }^{5}$ Program in Science, Technology and Society, University of Puget Sound, \\ 1500 North Warner Street, \\ Tacoma, WA 98416, USA \\ ${ }^{a}$ E-mail: kamalnandi1952@yahoo.co.in \\ ${ }^{b}$ E-mail: potapovaa@mail.ru \\ ${ }^{c}$ E-mail: izmailov.ramil@gmail.com \\ ${ }^{d}$ E-mail: amarjit.tamang1986@gmail.com \\ ${ }^{e}$ E-mail: jcevans@pugetsound.edu
}

\begin{abstract}
It is concluded in the literature that Ellis wormhole is unstable under small perturbations and would decay either to the Schwarzschild black hole or expand away to infinity. While this deterministic conclusion of instability is correct, we show that the Ellis wormhole reduces to Schwarzschild black hole only when the Ellis solution parameter $\gamma$ assumes a complex value $-i$. We shall then reexamine stability of Ellis and phantom wormholes from the viewpoint of local and asymptotic observers by using a completely different approach, viz., we adapt Tangherlini's nondeterministic, prequantal
\end{abstract}


statistical simulation about photon motion in the real optical medium to an effective medium reformulation of motions obtained via Hamilton's opticalmechanical analogy in a gravity field. A crucial component of Tangherlini's idea is the observed increase of momentum of the photons entering a real medium. We show that this fact has a heuristic parallel in the effective medium version of the Pound-Rebka experiment in gravity. Our conclusion is that there is a non-zero probability that Ellis and phantom wormholes could appear stable or unstable depending on the location of observers and on the values of $\gamma$, leading to the possibility of ghost wormholes (like ghost stars). The Schwarzschild horizon, however, would always appear certainly stable $(R=1, T=0)$ to observers regardless of their location. Phantom wormholes of bounded mass in the extreme limit $a \rightarrow-1$ are also shown to be stable just as the Schwarzschild black hole is. We shall propose a thought experiment showing that our non-deterministic results could be numerically translated into observable deterministic signatures of ghost wormholes.

PACS numbers: 04.20.-q, 04.25.-g, 04.40.-b

\section{Introduction}

About forty years ago, Tangherlini [1] using heuristic arguments derived the Fresnel reflectivity $(R)$ and transmissivity $(T)$ coefficients for a photon entering from vacuum into a homogeneous, isotropic, semi-infinite real medium. The method depends in part on the behavior of the momentum of light in ordinary refractive media, and in part on the assumption of a probabilistic condition at the surface where the photon impinges. And why today, after so many years, this work is recalled at all? The reason is that Tangherlini's approach could provide a new method of studying an issue of current interest, namely, the stability of static spherically symmetric solutions of general relativity. It would be of interest to explore the impact of Tangherlini's idea of "non-classical, prequantal statistical simulation" of reflectivity $(R)$ and transmissivity $(T)$ coefficients on the (in)stability of the Ellis [2] and phantom wormhole [3] solutions.

The static, spherically symmetric Ellis wormhole, independently derived also by Bronnikov [4], is an excellent example of a natural (as opposed to artificially assembled), everywhere regular, traversable wormhole solution of Einstein's equations sourced by a massless ghost scalar field that has a negative sign before the kinetic term ${ }^{1}$. González, Guzmán and Sarbach [5] have

\footnotetext{
${ }^{1}$ The solution should be appropriately termed as the Ellis-Bronnikov wormhole but
} 
shown that both the massless and massive wormholes are linearly unstable for the more general class of spherically symmetric perturbations, which do not necessarily vanish at the throat. The authors, in a subsequent paper [6], showed that Ellis wormholes are unstable also under non-linear perturbations such that the wormhole either expands away rapidly or collapses to a Schwarzschild black hole. The stability analysis has been extended by Bronnikov, Fabris and Zhidenko [7] to small radial perturbations of scalar-vacuum configurations with arbitrary external potential $V(\phi)$. They concluded the same instability. See also [8].

There is also an exception in the literature: Previously, Armendáriz-Picón [9] showed that massless Ellis wormhole and at least a non-zero measure set of massive Ellis wormholes are stable. But it is subsequently argued by González, Guzmán and Sarbach [5] that the linear stability analysis in [9] is "incomplete in the sense that it considers only a restricted class of perturbations where the areal radius of the wormhole throat is unperturbed and the perturbed scalar field vanishes on the throat. Such perturbations are somewhat artificial because one could imagine perturbing the scalar field by a small ingoing pulse which is supported away from the throat at some time $t=0$, say. As $t$ grows, this pulse travels towards the throat and since the wormhole metric is everywhere regular one expects it to reach and cross the throat at some finite time. On the other hand, requiring that the perturbed scalar field $(\delta \phi)$ vanishes at the throat corresponds to placing a mirror at the throat which reflects the scalar pulse." We shall take this quote as a guiding line and (in)stability of a wormhole will be determined by the probability of reflection of pulses by the wormhole throat.

The analysis in [5] uses perturbation of the scalar field by a small ingoing pulse of unidentified physical origin because from the mathematical viewpoint such identification is not mandatory. However, from the physical viewpoint, two issues could be raised: what causes the perturbation and who observes the resultant instability of the Ellis wormhole? To that end, we shall assume that the perturbation $\delta \phi$ of the scalar field $\phi$ is realistically caused by the motion of photons and matter particles. Further, we shall pay due consideration to the location of observers. In general relativity, both the issues are of paramount importance because motion of objects in the gravity field depends on their asymptotic velocities - for photons it is always

since it is more commonly known as Ellis wormhole, such as in Ref.[6], we keep to this common nomenclature in order to avoid confusion. 
unchangeably $c_{0}$, whereas for particles it can always be changed at will to any value less than $c_{0}$. Likewise, observations depend on the location that determines the scales and clocks of the observer for the measurement of an observable there. Since the master equation in [5] is expressed in the "coordinate" $(t, x, \theta, \varphi)$ language, the instability then is supposed to be observed by asymptotic observers, whose scales and clocks are unaffected by gravity 2 ,

The motivation of and the methodology for this work are as follows. Gravity is perceived as an effective refractive medium ${ }^{3}$ by different observers observing the photons travel towards the throat and reflected off or transmitted through it. It will be seen that the equations valid in the real medium remarkably resemble the equations derived in the effective medium, and supported by experiment in the latter. It is exactly this situation that motivates us to employ Tangherlini's probabilistic coefficients in the effective medium. It is further shown in [1] that, as a result of a certain cancellation in the statistical simulation, arising out of the independence of collisions, the coefficients are the same for one photon as for a large number of photons. This result is of importance for the interpretation of probabilistic coefficients at the wormhole throat. We shall extend these coefficients here to include also de Broglie matter waves.

We know that in a real medium (e.g., Bose-Einstein Condensate), it is quite possible to simulate novelties such as "laboratory optical black holes"

\footnotetext{
${ }^{2}$ Two types of quantities should be distinguished in a gravity field, hence by default in the effective optical medium (see Sec.3), which are: (i) "Coordinate" quantities (denoted here by primes and observed by asymptotic observers) are defined by scales and clocks unaffected by gravity, e.g., $c^{\prime}(\mathbf{r})=\left|\frac{d \mathbf{r}}{d t}\right|=\frac{c_{0}}{n(\mathbf{r})}$ [see Eq.(8)]. This is nothing but the exact equation [10] for Shapiro time delay $(\Delta t)$ measured by observers that are physically located at almost asymptotic locations, where proper scales are nearly equal to coordinate scales. Also, the familiar geodesic equations for planetary precession, light deflection etc are all expressed in coordinate language with the effects measured by observers stationed at asymptotic locations. (ii) "Proper" quantities (denoted here by tilde and observed by local observers) are defined by scales and clocks affected by gravity, e.g., the proper length $d L=\Phi^{-1}|d \mathbf{r}|$ and proper time $d \tau=\Omega d t$, so that $\widetilde{c}(\mathbf{r})=\left|\frac{d L}{d \tau}\right|=c_{0}$. (iii) In the absence of gravity or medium everywhere, we have what we call here "free space" quantities denoted without primes or tildes $(n=1)$ for which $c(\mathbf{r})=\left|\frac{d \mathbf{r}}{d t}\right|=c_{0}$.

${ }^{3}$ The effective refractive medium approach to gravity for light propagation was first pointed out by Eddington [11], an idea further developed by de Felice [12] and later extended to include both light and massive particle motions [13-18]. The works include WKB approximation [14], quantum phase [15], Fresnel drag coefficients [16] and cosmology [17]. Going a step further, even the rotational Kerr metric could be framed as a rotating medium with the force on a particle analogous to Lorentz force of electrodynamics [18].
} 
[19-25] or in the acoustic medium "laboratory acoustic black holes" [26-31] that mimic general relativistic ones. Our methodology here is to follow a reverse route, viz., start with general relativity, go to effective medium as an intermediate avatar, therein apply wisdom borrowed from real medium such as those of Tangherlini, and interpret the effects back in the realm of general relativity. That is, once we evaluate the non-deterministic coefficients in the effective medium, we can re-interpret them as chances of stability of the wormhole that lives in general relativity, and there will be no more need to talk of the intermediary effective medium.

The purpose of this paper therefore is to revisit the issue of stability from a physical viewpoint without manifestly needing any mathematical gauge fixing. We shall explore the extent to which a wormhole throat (Ellis and phantom) can reflect an ingoing disturbance, caused by the motion of a photon or a matter particle, observed by asymptotic and near-throat local observers. Following the argument in [5] quoted above, we shall assume that the probability of stability is determined by the probability of such reflection, that is, $R=1$, or equivalently $T=0$, imply certainty of stability since $R+T=1$. Rephrased in the spirit of [5], intermediate values of $(R, T)$ would mean the degree of uncertainty of reflection, hence uncertainty of (in)stability. To calculate $R$ and $T$, we shall employ a combination of two fundamental pre-quantum ideas: Tangherlini's probabilistic formulation [1] and Hamilton's optical-mechanical analogy applying it to motion in a gravity field. It turns out that there is a non-zero probability that Ellis and phantom wormholes could appear stable depending on the asymptotic speed of the particles as well as on the location of observers, leading to a new possibility of what we call here "ghost wormholes" (like ghost stars). We shall propose a thought experiment for observable deterministic signatures of ghost wormholes.

The paper is organized as follows: We outline in Sec.2, Tangherlini's formulation dealing with photon motion in real refractive media. Next, in Sec.3, we implement Hamilton's optical-mechanical analogy in the gravity field to develop the motion of de Broglie matter waves in the effective medium. Equations for photon motion follow as a special case. In Sec.4, we show that the momentum increase of particles entering the real medium has an exact parallel in the effective medium version of the Pound-Rebka experiment. We devote Sec.5 to a discussion of Ellis wormhole pointing out some of its interesting features and provide analytical support to the possibility of its collapse to Schwarzschild black hole. In Sec.6, we shall develop Tangherlini's coef- 
ficients for the motion of photon and matter de Broglie waves causing the perturbations. In Sec.7, we apply these coefficients to Schwarzschild black hole, Ellis wormhole and speculate on the possibility of ghost wormholes. Sec. 8 is devoted to phantom wormholes and Sec.9 describes a thought experiment. Conclusions are summarized in Sec.10. We shall take $8 \pi G=1$, speed of light in vacuum $c_{0}=1$, unless specifically restored. Signature convention is $(+---)$.

\section{Tangherlini's formulation}

Tangherlini's considerations tacitly assume scales and clocks unaffected by the medium and his ingredients are summarized below in (T1)-(T3):

(T1) Light is normally incident on a plane, semi-infinite, homogeneous, isotropic, non-absorbing real medium. If the photon is transmitted, the magnitude of its momentum $p^{\prime}$ in the medium is related to the magnitude of its momentum $p$ in free space by the equation

$$
p^{\prime}=n p
$$

where $n(\geq 1)$ is the index of refraction. This equation, which holds independently of the angle of incidence, is an experimental fact in the real medium. The prediction of the increase in momentum in the refractive medium has been verified to $15 \%$ accuracy by early experiments [32]. After a gap of about half a century, experiments by Jones [33], Jones and Richards [34], and by Ashkin and Dziedzic [35] confirmed the increase to a remarkable accuracy. Note that the de Broglie relation $p^{\prime} \lambda^{\prime}=p \lambda=$ constant (without Planck's constant) together with the reduction of wavelength in the real optical medium, $\lambda^{\prime}=\lambda / n$, which we shall soon verify in the effective medium, lead to Eq.(1); unprimed quantities $p$ and $\lambda$ refer to those in free space (absence of gravity or effective medium).

From a dynamical standpoint, the treatment of a photon may be based in the ray approximation on the following Hamiltonian, for negligible dispersion (see Eq.(A1) of Tangherlini [1]):

$$
H^{\prime}=\frac{c_{0}}{n(r)} p^{\prime}
$$

where $n=n(r)$ is a slowly varying refractive index for a real medium oriented so that the boundary is in the transverse plane.

We point out that Eq.(1) with $n=n(r)$ is an experimental fact supported by the effective medium version of the Pound-Rebka experiment of 
gravitational frequency shift (see Sec.4). It thus follows that in the effective medium, we need not be restricted by real medium constraints such as a constant $n$ or a sharp medium boundary.

(T2) The rate at which energy is transmitted to the real medium is proportional to the phase velocity of classical Hamiltonian mechanics:

$$
V_{\text {phase }}^{\prime}=\frac{c_{0}}{n(r)} .
$$

We shall show that this equation exactly holds in the effective medium as well and point out two other examples in footnote 4, where central equations in a real medium resemble those in the effective medium.

(T3) To avoid a deterministic passage of a photon across an impinging surface, Tangherlini [1] introduced a statistical method, which assumes that the probability for a photon to be found in a reflected or a transmitted mode on that surface can be calculated from an ensemble average. The ensemble consists of a large number of identical, widely separated replica of the media having the same refractive index. There is one ensemble representative for each incident photon. This condition, which does not involve Planck' s constant, introduces a kind of pre-quantum indeterminacy that leaves the motion of individual particles undetermined. The probability of reflection will be the fraction of the total number of particles that are observed to have been in the reflected mode, and likewise for the probability of the transmission.

Let $I$ denote the incident particle flux, let $I_{R}$ be the flux of reflected particles, and let $I_{T}$ be the flux of transmitted particles such that

$$
I_{R}+I_{T}=I .
$$

In accordance with standard notation, the ratios $R=I_{R} / I$ and $T=I_{T} / I$ are taken to define the probabilities of reflection and transmission respectively. These probabilities satisfy the so-called conservation of probability condition

$$
R+T=1
$$

Equating the average rate of energy delivered to the ensemble member in reflected and transmitted modes, Tangherlini [1] derives the coefficients:

$$
R=\frac{(n-1)^{2}}{(n+1)^{2}}, T=\frac{4 n}{(n+1)^{2}},
$$


that remain invariant under $n \rightarrow 1 / n$. These coefficients exactly resemble those that are obtained by solving Schrödinger equation for a certain potential [36]. We shall be using Eqs.(6) in the sequel.

\section{Optical-mechanical analogy: Motion of particles}

We shall implement Hamilton's optical-mechanical analogy in the gravity field to arrive at the motion of particles and light that exactly reproduces motions of massive (and massless) particles already otherwise known, for example, in the Schwarzschild gravity field $[10,14]$. For our purpose, we shall consider the generic form of a static, spherically symmetric metric field in isotropic coordinates

$$
d s^{2}=\Omega^{2}(\mathbf{r}) c_{0}^{2} d t^{2}-\Phi^{-2}(\mathbf{r})\left[d r^{2}+r^{2}\left(d \theta^{2}+\sin ^{2} \theta d \varphi^{2}\right)\right],
$$

where $\Omega(\mathbf{r})$ and $\Phi(\mathbf{r})$ are arbitrary metric functions of spatial coordinates $\mathbf{r} \equiv(r, \theta, \varphi)$ or $\left(x_{1}, x_{2}, x_{3}\right)$. The coordinate phase speed of light $c^{\prime}(\mathbf{r})$, observed by asymptotic observers, is determined by the condition that the geodesic be null $\left(d s^{2}=0\right)$ giving

$$
c^{\prime}(\mathbf{r})=\left|\frac{d \mathbf{r}}{d t}\right|=c_{0} \Phi(\mathbf{r}) \Omega(\mathbf{r})=\frac{c_{0}}{n(\mathbf{r})} .
$$

Thus the effective index of refraction for light in the gravitational field is:

$$
n(\mathbf{r})=\Phi^{-1} \Omega^{-1}
$$

This index of refraction may be used in any formulation of geometrical optics using Fermat's principle:

$$
\delta \int_{\mathbf{x}_{1}}^{\mathbf{x}_{2}} n|d \mathbf{r}|=0
$$

where $\delta$ represents a variation of the integral produced by varying the path between two fixed points $\mathbf{x}_{1}$ and $\mathbf{x}_{2}$ in the three-dimensional Euclidean space and $|d \mathbf{r}|$ is the element of the path of integration.

In general relativity, the orbits of massive test particles are obtained by requiring that they be geodesics:

$$
\delta \int_{\mathbf{x}_{1}, t_{1}}^{\mathbf{x}_{2}, t_{2}} m_{0} d s=0
$$


where $\left(\mathbf{x}_{1}, t_{1}\right)$ and $\left(\mathbf{x}_{2}, t_{2}\right)$ are two fixed points in spacetime, $m_{0}$ is the rest mass of the test particle. Using Eqs.(7) and (9), the above can be rewritten in the form of Hamilton's variational principle

$$
\delta \int_{t_{1}}^{t_{2}} L^{\prime}\left(x_{i}, V_{i}^{\prime}\right) d t=0,
$$

where the effective Lagrangian is:

$$
L^{\prime}\left(x_{i}, V_{i}^{\prime}\right) \equiv-m_{0} c_{0}^{2} \Omega\left[1-\frac{n^{2} V^{\prime 2}}{c_{0}^{2}}\right]^{1 / 2} .
$$

In the above expression, the coordinate velocity $V^{\prime}$ is defined by $V^{\prime 2}=\sum_{i=1}^{3} V_{i}^{\prime 2}$ and $V_{i}^{\prime} \equiv\left(\frac{d \mathbf{r}}{d t}\right), \mathbf{r} \equiv\left(x_{1}, x_{2}, x_{3}\right)$. Similarly, the canonical coordinate momenta of a particle in the gravity field are

$$
p_{i}^{\prime}=m_{0} \Omega n^{2}\left[1-\frac{n^{2} V^{\prime 2}}{c_{0}^{2}}\right]^{-1 / 2} V_{i}^{\prime},
$$

where the primed notation $p^{\prime}$ is used only to be in conformity with (T1). The effective Hamiltonian is

$$
H^{\prime}=m_{0} c_{0}^{2} \Omega\left[1-\frac{n^{2} V^{\prime 2}}{c_{0}^{2}}\right]^{-1 / 2},
$$

\footnotetext{
${ }^{4}$ Here are the two other examples we promised in the preceding section, where the central equations in a real medium resemble those in the present effective medium: (i) Starting from the wave equation in a non-uniformly moving real fluid with refractive index $n$, Leonhardt and Piwnicki [21] derive the Lagrangian and the Hamiltonian for a light ray as observed by a lab observer. From the action principle, they arrive at a completely geometrical picture of ray optics in a moving medium. Light rays are geodesic lines with respect to the Gordon metric, which, in the comoving frame of the real fluid element reads: $d s^{2}=\frac{c_{0}^{2}}{n^{2}} d t^{2}-d \mathbf{r}^{2}$ and the Lagrangian reads [21]: $L^{\prime}\left(x_{i}, V_{i}^{\prime}\right) \equiv-m_{0} c_{0}^{2} \times \frac{1}{n} \times\left[1-\frac{n^{2} V^{\prime 2}}{c_{0}^{2}}\right]^{1 / 2}$, where $V^{\prime}$ is the particle coordinate speed. The Lagrangian for a massive particle in the comoving frame derived in this paper is [Eq.(13)]: $L^{\prime}\left(x_{i}, V_{i}^{\prime}\right) \equiv-m_{0} c_{0}^{2} \times \Omega \times\left[1-\frac{n^{2} V^{\prime 2}}{c_{0}^{2}}\right]^{1 / 2}$. Now note that the Gordon metric with $n$ as the real medium index, can be obtained formally from the metric (7) above simply by putting $\Phi=1, \Omega=\frac{1}{n}$ so that $n=\Phi^{-1} \Omega^{-1}$. Clearly, the $n$ in Eq.(13) has a different origin: it derives from the equivalent medium. This notwithstanding, one finds that the Lagrangians are exactly the same. (ii) The dispersion relation for light in the comoving frame derived in [21] is $\omega^{\prime 2}-c_{0}^{2} k^{\prime 2}+\left(n^{2}-1\right) \omega^{\prime 2}=0$. This is precisely the same as that following from Eq.(69) with $m_{0}=0$ for light. See [16] for details.
} 
or expressed in terms of momenta

$$
H^{\prime}=m_{0} c_{0}^{2}\left[\Omega^{2}+\frac{p^{2}}{n^{2} m_{0}^{2} c_{0}^{2}}\right]^{1 / 2},
$$

which is a constant of motion. Its value may be calculated from Eq.(15) if the particle coordinate speed $V^{\prime}$ is known at one point on the path. Eq.(15) gives the particle coordinate speed in the medium as

$$
V^{\prime}(\mathbf{r})=\frac{c_{0}}{n}\left[1-\frac{m_{0}^{2} c_{0}^{4} \Omega^{2}}{H^{\prime 2}}\right]^{1 / 2} .
$$

For the motion of a massive particle inside the medium, we have from Hamilton's principle, Eq.(12), the Jacobi's form of Maupertuis's principle, which is, using Eq.(14):

$$
\delta \int_{\mathbf{x}_{1}}^{\mathbf{x}_{2}} p^{\prime}|d \mathbf{r}|=0
$$

where now the path of integration through three-dimensional space is varied, subject to conservation of energy, between two fixed points in space, $\mathbf{x}_{1}$ and $\mathbf{x}_{2}$, but the times at the end points need not be held fixed. From Eqs.(14) and (15), we have

$$
p_{i}^{\prime}=H^{\prime} n^{2} V_{i}^{\prime} / c_{0}^{2} .
$$

Specializing to photon motion $\left(m_{0}=0\right)$, we see that it has the phase speed [it is also the group speed, see Eq.(72)] in the effective medium

$$
V^{\prime}(\mathbf{r})=\frac{c_{0}}{n}
$$

which leads to

$$
H^{\prime}=\frac{c_{0} p^{\prime}}{n},
$$

and we have here rederived Tangherlini's Eqs.(2) and (3), as promised. Hence for a free photon at infinity $(n=1)$

$$
H=c_{0} p .
$$

Using the value of $p^{\prime}$ from Eq.(19), Eq.(18) can be written as

$$
\delta \int_{\mathbf{x}_{1}}^{\mathbf{x}_{2}} \frac{H^{\prime} n^{2} V^{\prime}}{c_{0}^{2}}|d \mathbf{r}|=0 .
$$


From this variational principle follows the exact general-relativistic equation of motion for a massive particle (see for details, Ref.[14]):

$$
\frac{d^{2} \mathbf{r}}{d A^{2}}=\nabla\left(\frac{1}{2} n^{4} V^{\prime 2}\right),
$$

which has precisely the form of Newton's second law for the "potential" $-\frac{1}{2} n^{4} V^{\prime 2}$. The independent variable $A$ playing the role of time in Eq.(24) is the optical action, defined by

$$
\left|\frac{d \mathbf{r}}{d A}\right|=n^{2} V^{\prime}, d A=\frac{d t}{n^{2}} .
$$

For photon, using Eq.(20) for $V^{\prime}$, the path Eq.(24) yields

$$
\frac{d^{2} \mathbf{r}}{d A^{2}}=\nabla\left(\frac{1}{2} n^{2} c_{0}^{2}\right)
$$

We see that $-\frac{1}{2} n^{2} c_{0}^{2}$ plays the role of "potential" for the massless particle. Likewise, we can rewrite Eq.(24) as

$$
\frac{d^{2} \mathbf{r}}{d A^{2}}=\nabla\left(\frac{1}{2} N^{2} c_{0}^{2}\right),
$$

such that $N\left(\equiv n^{2} V^{\prime} / c_{0}\right)$ plays the role of index of refraction for the massive particle. But since $V^{\prime}=|d \mathbf{r} / d t|$, it follows that along the trajectory, Eq. (25) holds. The "potential" on the right hand side and the "acceleration" on the left in Eqs.(26), (27) are the optical versions describing the mechanical motion of photon and massive particles respectively.

With the above results at hand, we shall now introduce de Broglie waves of a massive particle into the medium with index of refraction. In the geometrical-optics limit, it is possible to speak of a ray, which is the trajectory of the point-particle, that is, Maupertuis principle for mechanics (18) is identical with the Fermat's principle for ray optics (10). This is the essence of Hamilton's optical-mechanical analogy implemented later by de Broglie in his wave-particle duality. The ray will be the path satisfying Fermat's principle, which we write in the form:

$$
\delta \int_{\mathbf{x}_{1}}^{\mathbf{x}_{2}} k^{\prime}|d \mathbf{r}|=0,
$$


where $k^{\prime}(\mathbf{r})$ is the wave number.

Reasoning in exactly the same way as would de Broglie, the paths predicted by Eq. (23) and Eq. (28) will be the same if the two integrands are the same functions of the spatial coordinates. However, they may differ by a multiplicative factor $f$. Thus we require

$$
\frac{H^{\prime} n^{2} V^{\prime}}{c_{0}^{2}}=f k^{\prime} .
$$

Going over to the free space limit, the above yields $m_{0} V^{\prime} \gamma=f k^{\prime}$ and this will be the de Broglie relation for a relativistic free particle only if $f=\hbar=h / 2 \pi$, the Planck constant ${ }^{5}$. Thus we have the momentum of the de Broglie waves as

$$
\frac{H^{\prime} n^{2} V^{\prime}}{c_{0}^{2}}=\hbar k^{\prime},
$$

which leads to the coordinate wavelength of the de Broglie waves in the effective medium as

$$
\lambda^{\prime}=\frac{h c_{0}^{2}}{H^{\prime} n^{2} V^{\prime}}
$$

Using Eq.(17) for $V^{\prime}(\mathbf{r})$, the above can be rewritten as

$$
\lambda^{\prime}=\frac{h c_{0}}{n H^{\prime}\left[1-\frac{m_{0}^{2} c_{0}^{4} \Omega^{2}}{H^{\prime 2}}\right]^{1 / 2}},
$$

that, in turn, yields

$$
\lambda^{\prime} n\left[1-\frac{m_{0}^{2} c_{0}^{4} \Omega^{2}}{H^{\prime 2}}\right]^{1 / 2}=\frac{h c_{0}}{H^{\prime}}=\text { constant. }
$$

Thus the wave-optics rule for massive particles may be expressed as

$$
\lambda^{\prime} N=\text { constant, }
$$

where the index of refraction $N$ for the de Broglie waves of massive particles in the medium is given by

$$
N=n\left[1-\frac{m_{0}^{2} c_{0}^{4} \Omega^{2}}{H^{\prime 2}}\right]^{1 / 2} .
$$

\footnotetext{
${ }^{5}$ To be in line with the pre-quantal indeterminacy of Tangherlini, identifying the constant $f$ with $\hbar$ is not mandatory. However, we have introduced matter de Broglie waves using $\hbar$ in a semiclassical manner but there is no role for it as it cancels out in the relevant expressions that follow [see, e.g., Eq.(37), in which $\hbar$ cancels out between $p^{\prime}$ and $H^{\prime}$ ].
} 
This can be rewritten as

$$
N=\frac{n^{2} V^{\prime}}{c_{0}} .
$$

With the help of Eq.(17), this can be further rewritten as

$$
N=\frac{c_{0} p^{\prime}}{H^{\prime}} .
$$

Eqs.(17), (34) and (37) are the generalized equations applicable to matter de Broglie waves.

Let us specialize to photon motion in the effective medium for which we put $m_{0}=0, N=n$ in Eqs.(17), (34) and (37) and collect the results in one place:

$$
\begin{aligned}
V_{\text {phase }}^{\prime} & =c^{\prime}(r)=\frac{c_{0}}{n(r)}, \\
\lambda^{\prime} n(r) & =\text { constant }=\lambda, \\
H^{\prime} & =\frac{c_{0}}{n(r)} p^{\prime}=\text { constant }=c_{0} p .
\end{aligned}
$$

Directly from Eq.(40), we get

$$
p^{\prime}=n(r) p,
$$

which is just Tangherlini's Eq.(1) for photon motion. All the above coordinate relations are the foundation of Tangherlini's formulation [1]. Asymptotic observers measure the index $n$ and photon momentum $p^{\prime}$ connected by Eq.(41) and wavelength $\lambda^{\prime}$ according to Eq.(39). The observers inside the medium measure proper wave length $\widetilde{\lambda}$ defined by $\widetilde{\lambda}=\lambda^{\prime} \Phi^{-1}$, and so Eq.(39) changes to

$$
\widetilde{\lambda}(n \Phi)=\widetilde{\lambda} \widetilde{n}(r)=\text { constant }=\lambda .
$$

Using de Broglie relations inside the medium, $\widetilde{p}=\hbar / \widetilde{\lambda}$ and $p=\hbar / \lambda$ in Eq.(42), when $\hbar$ cancels out, we get

$$
\widetilde{p}=\widetilde{n}(r) p,
$$

where $\widetilde{n}>1$. This is the proper version of Eq.(1) inside the effective medium for ingoing photons that is supported by the Pound-Rebka experiment as shown below. 


\section{Pound-Rebka experiment}

It is shown above that Tangherlini's Eqs.(1)-(3) are just Eqs.(38), (40), (41) in the effective medium. The question now is: like the experiments supporting Eq.(1) in a real optical medium, is there any experiment confirming the validity of Eq.(43), or the increase of light momentum inside the effective medium? The answer is yes, and it does not seem widely recognized that it is just the medium version of the famous Pound-Rebka frequency shift experiment [37], although this version is purely heuristic based on the mathematical similitude of the relevant equations.

Let us recall the set-up, where a pulse of light falls to Earth's surface from the roof of a building of height $h_{0}$. For a Schwarzschild gravity of mass $M$ describing the Earth's gravity, we have

$$
n_{\mathrm{Sch}}(r)=\frac{\left(1+\frac{M}{2 r}\right)^{3}}{\left(1-\frac{M}{2 r}\right)} \simeq 1+\frac{2 M}{r} .
$$

Accordingly, using Eq.(41), the coordinate relations (primes replaced by suffixes for transparency) are

$$
\frac{p_{\mathrm{E}}}{p_{\mathrm{R}}}=\frac{\lambda_{\mathrm{R}}}{\lambda_{\mathrm{E}}}=\frac{n_{\mathrm{E}}}{n_{\mathrm{R}}}
$$

where the suffix R refers to quantities at the roof, and E refers to those on the Earth, $r_{\mathrm{E}}$ is the radius of the Earth. (The isotropic radius $r_{\mathrm{E}}$ is far greater than Earth's black hole radius $\frac{G M}{2 c_{0}^{2}}$ of a few centimeters, so Earth's radius in "standard" coordinates would be very nearly the same as $r_{\mathrm{E}}$, differing by those few centimeters). Note that both the events of emission and reception are taking place inside the effective medium so that, using Eqs.(42) and (43), we get

$$
\frac{\widetilde{p}_{\mathrm{E}}}{\widetilde{p}_{\mathrm{R}}}=\frac{\widetilde{\lambda}_{\mathrm{R}}}{\widetilde{\lambda}_{\mathrm{E}}}=\frac{n_{\mathrm{E}} \Phi_{\mathrm{E}}}{n_{\mathrm{R}} \Phi_{\mathrm{R}}},
$$

where $n \Phi>1$. Neglecting $\left(h_{0} / r_{\mathrm{E}}\right)^{2}$ and higher orders, we get

$$
\begin{aligned}
& \frac{n_{\mathrm{E}}}{n_{\mathrm{R}}} \simeq\left(1+\frac{2 M}{r_{\mathrm{E}}}\right) /\left(1+\frac{2 M}{r_{\mathrm{E}}+h_{0}}\right) \simeq 1+\frac{2 M}{r_{\mathrm{E}}^{2}} h_{0}, \\
& \frac{\Phi_{\mathrm{E}}}{\Phi_{\mathrm{R}}} \simeq 1-\frac{M}{r_{\mathrm{E}}^{2}} h_{0} .
\end{aligned}
$$


Hence, to first order in $M$,

$$
\frac{\widetilde{p}_{\mathrm{E}}}{\widetilde{p}_{\mathrm{R}}}=\frac{\widetilde{\lambda}_{\mathrm{R}}}{\widetilde{\lambda}_{\mathrm{E}}}=1+\frac{M}{r_{\mathrm{E}}^{2}} h_{0} .
$$

The second equality has been very accurately confirmed by the Pound-Rebka experiment in gravity, which now implies that $\widetilde{p}_{\mathrm{E}}>\widetilde{p}_{\mathrm{R}}$. A similar experiment with matter de Broglie waves testing the equation

$$
\frac{\widetilde{p}_{\mathrm{E}}}{\widetilde{p}_{\mathrm{R}}}=\frac{\widetilde{\lambda}_{\mathrm{R}}}{\widetilde{\lambda}_{\mathrm{E}}}=\frac{N_{\mathrm{E}} \Phi_{\mathrm{E}}}{N_{\mathrm{R}} \Phi_{\mathrm{R}}},
$$

where $N \Phi>1$, has also been suggested in the literature [14].

Therefore, what is known as the gravitational frequency shift could be interpreted as the momentum increase of the ingoing pulse in the effective medium. One sees that the effective medium behaves exactly like a real medium in this regard. The premise for the application of Tangherlini's approach to wormholes is now ready at hand. Accordingly, in the next section we shall briefly discuss Ellis wormhole and thereafter develop the reflection and transmission coefficients for photon and de Broglie waves. Later we shall apply them also to phantom wormhole.

\section{Ellis wormhole and Schwarzschild black hole}

This wormhole is a solution of Einstein's field equations

$$
R_{\mu \nu}=\varepsilon \phi_{, \mu} \phi_{, \nu}
$$

where $\phi_{, \mu} \equiv \frac{\partial \phi}{\partial x^{\mu}}, \phi$ being a massless scalar field. Choosing $\varepsilon=-1$, which represents the ghost regime, the static spherically symmetric massive Ellis wormhole in isotropic coordinates can be written as

$$
d \tau^{2}=\Omega^{2}(r) d t^{2}-\Phi^{-2}(r)\left[d r^{2}+r^{2}\left(d \theta^{2}+\sin ^{2} \theta d \varphi^{2}\right)\right],
$$

where

$$
\begin{aligned}
\Omega^{2}(r) & =\exp \left[2 \epsilon+4 \gamma \tan ^{-1}(r / B)\right] \\
\Phi^{-2}(r) & =\left(1+\frac{B^{2}}{r^{2}}\right)^{2} \exp \left[2 \zeta-4 \gamma \tan ^{-1}(r / B)\right], \\
\phi(r) & =4 \lambda \tan ^{-1}(r / B),
\end{aligned}
$$


with the constraint $2 \lambda^{2}=1+\gamma^{2}$ following from the field Eq.(51), $B$ and $\gamma$ being arbitrary constants. Asymptotic flatness requires that $\epsilon=-\pi \gamma$ and $\zeta=\pi \gamma$. The spacetime has no horizon, is twice asymptotically flat and is a traversable, everywhere regular natural wormhole (as opposed to artificially assembled ones) with the throat appearing at the isotropic coordinate radius $r_{\text {th }}=\frac{M}{2 \gamma}\left[\gamma+\sqrt{1+\gamma^{2}}\right]$.

To put it into a familiar form, transform the radial coordinate $r \rightarrow \ell$ by $\ell=r-\frac{B^{2}}{r}$, where $\ell \in(-\infty, \infty)$ corresponding to $r \in(0, \infty)$. Redefining $B=m / 2$, and using the identity

$$
2 \tan ^{-1}\left(\frac{\ell+\sqrt{\ell^{2}+m^{2}}}{m}\right) \equiv \frac{\pi}{2}+\tan ^{-1}\left(\frac{\ell}{m}\right),
$$

we see that the solution set (53)-(55) transforms exactly to the form of Ellis class III wormhole solution (the Ellis wormhole) [2]:

$$
\begin{aligned}
d \tau_{\text {Ellis }}^{2} & =F d t^{2}-F^{-1}\left[d \ell^{2}+\left(\ell^{2}+m^{2}\right)\left(d \theta^{2}+\sin ^{2} \theta d \varphi^{2}\right)\right] \\
F & =\exp \left[-\pi \gamma+2 \gamma \tan ^{-1}\left(\frac{\ell}{m}\right)\right] \\
\phi & =\lambda\left[\pi+2 \tan ^{-1}\left(\frac{\ell}{m}\right)\right]
\end{aligned}
$$

with the constraint $2 \lambda^{2}=1+\gamma^{2}$ remaining the same. Note that $\ell$ is not the proper radial distance, the latter being given by $L=\int F^{-1 / 2} d \ell$. The wormhole has manifestly two asymptotically flat regions, one with positive mass $M(=m \gamma)$ and the other with negative mass $-M e^{\pi \gamma}$, on either side of a regular throat (minimal area radius) at $\ell_{\mathrm{th}}=M$. A single extended coordinate chart $(t, \ell, \theta, \varphi)$ across the regular throat now covers both the sides. The same solution is used in [5] that can be seen by renaming $\ell \equiv x$, $m \equiv b$ and their constants as $\gamma_{1}=\gamma, \gamma_{0}=-\frac{\pi}{2} \gamma, \Phi_{0}=\lambda \pi, \Phi_{1}=2 \lambda$. The constraint $2 \lambda^{2}=1+\gamma^{2}$ is just their equation: $\Phi_{1}^{2}=2\left(1+\gamma_{1}^{2}\right)$.

Using the Ellis wormhole metric functions $\Omega$ and $\Phi$ from Eqs.(53),(54) and identifying the constant as $B=\frac{M}{2 \gamma}$, we see that the equivalent refractive 
index for photon motion perceived by asymptotic observers is

$$
\begin{aligned}
n(r) & =\Phi^{-1} \Omega^{-1}=\left(1+\frac{M^{2}}{4 r^{2} \gamma^{2}}\right) \exp \left[2 \pi \gamma-4 \gamma \tan ^{-1}\left(\frac{2 r \gamma}{M}\right)\right] \\
& \simeq 1+\frac{2 M}{r}+\left(2+\frac{1}{4 \gamma^{2}}\right)\left(\frac{M}{r}\right)^{2}+\left(\frac{1+4 \gamma^{2}}{3 \gamma^{2}}\right)\left(\frac{M}{r}\right)^{3}+\ldots
\end{aligned}
$$

where $M=m \gamma$ is the Keplerian mass observed by the asymptotic observers. This expansion does not coincide with the expansion of refractive index for the Schwarzschild gravity for the same mass $M$, viz.,

$$
n_{\mathrm{Sch}}(r) \simeq 1+\frac{2 M}{r}+\left(\frac{7}{4}\right)\left(\frac{M}{r}\right)^{2}+\left(\frac{M}{r}\right)^{3}+\ldots
$$

for any real $\gamma$. Here we recall that a collapse to a Schwarzschild black hole is possible from the Ellis class I solution [2] (naked singularity, not a wormhole) because there is a smooth passage from it to the black hole under the trivial choice $\gamma=1$ (the so called no-hair theorem). In its vacuum Brans-Dicke representation of the Ellis class I solution, the scalar field is radiated away in the Oppenheimer-Snyder collapse resulting into a black hole identical to that in general relativity, as has been shown by Scheel, Shapiro and Teukolsky [38].

The passage from the Ellis wormhole to Schwarzschild black hole happens only if one puts $\gamma=-i$ in Eq.(61). Therefore, the collapse to the latter might at best seem fortuitous. That however need not be the case although the route is by no means trivial. Consider the metric (53-55) under inversion and Wick-like rotation: $r \rightarrow \frac{1}{r}, B \rightarrow \frac{i}{B}, \gamma=-i$. With $B=\frac{M}{2 \gamma}$, we obtain $\Omega^{2}(r)=e^{-4 \tanh ^{-1}\left(\frac{M}{2 r}\right)}$. Using further the identity $e^{\tanh ^{-1}(x)} \equiv \frac{1}{2} \ln \left(\frac{1+x}{1-x}\right)$, we get $\Omega^{2}(r)=\left(\frac{1-\frac{M}{2 r}}{1+\frac{M}{2 r}}\right)^{2}$ and $\Phi^{-2}(r)=\left(1+\frac{M}{2 r}\right)^{4}$, which is just the Schwarzschild metric in the isotropic form. And of course the scalar field $\phi=0$, as it should be, and the isotropic coordinate radius of the throat $r_{\text {th }}=\frac{M}{2 \gamma}\left[\gamma+\sqrt{1+\gamma^{2}}\right]$ converts to coordinate horizon: $r_{\text {hor }}=\frac{M}{2}$. This derivation provides a completely analytic reason as to why González, Guzmán and Sarbach [6] get a "strong indication" in their numerical simulation of the Ellis wormhole collapsing to the Schwarzschild black hole.

\section{Reflection and Transmission coefficients}
(a) Light pulse: 
The throat of the Ellis wormhole occurs at the minimum of the areal radius $R(r)=r \Phi^{-1}(r)$, which gives

$$
r_{\mathrm{th}}=\frac{M}{2 \gamma}\left[\gamma+\sqrt{1+\gamma^{2}}\right]
$$

The coefficients for the ingoing photon pulse at the throat as observed by asymptotic observers (a.o.) are given, using Eqs.(6) and (60) with $n$, by:

$$
\begin{aligned}
R_{\text {photon }}^{\text {a.o. }} & =\frac{\left[n\left(r_{\mathrm{th}}\right)-1\right]^{2}}{\left[n\left(r_{\mathrm{th}}\right)+1\right]^{2}}, \\
T_{\text {photon }}^{\text {a.o. }} & =\frac{4 n\left(r_{\mathrm{th}}\right)}{\left[n\left(r_{\mathrm{th}}\right)+1\right]^{2}} .
\end{aligned}
$$

The coefficients for the ingoing photon pulse at the throat as observed by near-throat local observers (l.o.) are given, using Eqs.(6) and (60) with $\tilde{n}$ $(=n \Phi)$ by:

$$
\begin{aligned}
& \widetilde{R}_{\text {photon }}^{\text {l.o. }}=\frac{\left[\widetilde{n}\left(r_{\mathrm{th}}\right)-1\right]^{2}}{\left[\widetilde{n}\left(r_{\mathrm{th}}\right)+1\right]^{2}}, \\
& \widetilde{T}_{\text {photon }}^{\text {loo. }}=\frac{4 \widetilde{n}\left(r_{\mathrm{th}}\right)}{\left[\widetilde{n}\left(r_{\mathrm{th}}\right)+1\right]^{2}} .
\end{aligned}
$$

(b) de Broglie matter waves:

The phase velocity of de Broglie waves is $v_{\text {phase }}^{\prime}=\frac{\omega^{\prime}}{k^{\prime}}=\frac{H^{\prime}}{p^{\prime}}$, or, with use of Eq.(37),

$$
v_{\text {phase }}^{\prime}=\frac{c_{0}}{N},
$$

a relation which demonstrates once again that $N$ is playing its desired role as an index of refraction for de Broglie matter waves. Inserting $p^{\prime}=\hbar k^{\prime}$ and $H^{\prime}=\hbar \omega^{\prime}$ into the square of Eq.(16), we obtain

$$
\hbar^{2} \omega^{\prime 2}=m_{0}^{2} c_{0}^{4} \Omega^{2}+\frac{c_{0}^{2} \hbar^{2} k^{\prime 2}}{n^{2}} .
$$

Differentiating with respect to $k^{\prime}$, we obtain

$$
\frac{\omega^{\prime}}{k^{\prime}} \frac{d \omega^{\prime}}{d k^{\prime}}=\frac{c_{0}^{2}}{n^{2}}, \text { or, } v_{\text {phase }}^{\prime} v_{\text {group }}^{\prime}=\frac{c_{0}^{2}}{n^{2}},
$$


where $v_{\text {group }}^{\prime}=\frac{d \omega^{\prime}}{d k^{\prime}}$ is the group velocity of the de Broglie waves ${ }^{6}$. Using Eq.(70), we get

$$
v_{\text {group }}^{\prime}=\frac{c_{0} N}{n^{2}} .
$$

For photons, $N=n$ and so

$$
V_{\text {phase }}^{\prime}=V_{\text {group }}^{\prime}=\frac{c_{0}}{n} .
$$

This is the same as Eq.(38). Substituting the expression for $N$ from Eq. (36) in Eq.(71), we see that

$$
v_{\text {group }}^{\prime}=V^{\prime} \text {. }
$$

Thus, $V^{\prime}$ defined by Eq.(17) is the group velocity of de Broglie matter waves. In the asymptotic limit $r \rightarrow \infty$, we have $n=1$, and the free particle has $V^{\prime}=V=$ constant so that from Eq.(36), we find the generic result

$$
N_{\infty}=\frac{V}{c_{0}} \equiv \beta
$$

Using $H^{\prime}=h \nu^{\prime}, \lambda^{\prime} \nu^{\prime}=c^{\prime}=\frac{c_{0}}{n}$, and introducing the Compton wavelength of the particle by $\lambda_{\mathrm{C}}=\frac{h}{m_{0} c_{0}}$, we can rewrite Eq.(35) as

$$
N(r)=n(r)\left[1-\left(\frac{\lambda^{\prime}}{\lambda_{\mathrm{C}}}\right)^{2} \Phi^{-2}(r)\right]^{1 / 2} .
$$

From Eq.(34), we get

$$
\lambda^{\prime} N=\lambda N_{\infty}
$$

Using the de Broglie relations $p^{\prime}=\hbar / \lambda^{\prime}$ and $p=\hbar / \lambda$ in Eq.(76), where $\hbar$ cancels out, we get

$$
p^{\prime}=\frac{N(r)}{\beta} p=\bar{N}(r) p, \bar{N}(r)=\frac{N(r)}{\beta}>1
$$

${ }^{6}$ Observers situated at infinity see that near the horizon, $n \rightarrow \infty$ so that $v_{\text {phase }}^{\prime}, v_{\text {group }}^{\prime} \rightarrow$ 0 , both for light and matter de Broglie waves, in perfect accordance with general relativistic scenario. It is exactly here that the conditions for laboratory "optical black holes" required by Leonhardt and Piwnicki [21,22] and Hau et al. [20] are met most naturally, that is, extremely low group velocity or high refractive index. Group velocity of light as low as 17 meters/sec in Bose-Einstein condensate has been attained in the laboratory [20]. See also [23]. In this respect, optical and gravitational black holes look much similar. 
at $r \geq r_{\text {th }}$ for wormholes. This $\bar{N}$ generalizes Eq.(41) to massive particles, where $c^{\prime}=c_{0} / \bar{N}(r)$. The expressions of the coefficients for the ingoing de Broglie waves as observed by asymptotic observers (a.o.) now become

$$
\begin{aligned}
\bar{R}_{\mathrm{dB}}^{\text {a.o. }} & =\frac{\left[\bar{N}\left(r_{\mathrm{th}}\right)-1\right]^{2}}{\left[\bar{N}\left(r_{\mathrm{th}}\right)+1\right]^{2}}, \\
\bar{T}_{\mathrm{dB}}^{\text {a.o. }} & =\frac{4 \bar{N}\left(r_{\mathrm{th}}\right)}{\left[\bar{N}\left(r_{\mathrm{th}}\right)+1\right]^{2}} .
\end{aligned}
$$

The observers inside the medium measure proper wave length $\tilde{\lambda}$ defined by $\widetilde{\lambda}=\lambda^{\prime} \Phi^{-1}$, and so Eq.(76) changes to

$$
\widetilde{\lambda}(\Phi N)=\tilde{\lambda} \widetilde{N}(r)=\text { constant }=\lambda N_{\infty},
$$

where

$$
\widetilde{N}(r)=\Phi(r) N(r)=\Phi(r) n(r)\left[1-\left(\frac{\tilde{\lambda}}{\lambda_{\mathrm{C}}}\right)^{2}\right]^{1 / 2} .
$$

Using de Broglie relations inside the medium, $\widetilde{p}=\hbar / \widetilde{\lambda}$ and $p=\hbar / \lambda$ in Eq.(80), where again $\hbar$ cancels out, we get

$$
\widetilde{p}=\frac{\widetilde{N}(r)}{\beta} p=\widehat{N}(r) p, \widehat{N}(r)=\frac{\widetilde{N}(r)}{\beta}=\frac{\Phi(r) N(r)}{\beta}>1,
$$

at $r \geq r_{\text {th }}$ for wormholes. This is the proper language version of Eq.(1) in the effective medium for ingoing massive particles. Accordingly, the expressions of the coefficients for the ingoing de Broglie waves as observed by near-throat local observers (l.o.) now become

$$
\begin{aligned}
\widehat{R}_{\mathrm{dB}}^{\mathrm{loo}} & =\frac{\left[\widehat{N}\left(r_{\mathrm{th}}\right)-1\right]^{2}}{\left[\widehat{N}\left(r_{\mathrm{th}}\right)+1\right]^{2}}, \\
\widehat{T}_{\mathrm{dB}}^{\text {lo. }} & =\frac{4 \widehat{N}\left(r_{\mathrm{th}}\right)}{\left[\widehat{N}\left(r_{\mathrm{th}}\right)+1\right]^{2}} .
\end{aligned}
$$


Four pairs of equations (64,65), (66,67) and (78,79), (83,84) are the coefficients that we have been looking for. Looking at them, we see that except for the first pair, the formulation of other three pairs is of hybrid nature as they have one leg in the effective medium defined by the index $n$ and the other in the metric function $\Phi$. For our purposes, it is enough to treat all of the equation pairs as merely some functions of $r$ and evaluate the coefficients for different observers in different metrics.

For applying the last two pairs of formulas, it is necessary to compare the two wavelengths $\lambda^{\prime}$ and $\lambda_{\mathrm{C}}$ appearing in them. The coordinate de Broglie wavelength $\lambda^{\prime}$ is related to a particle's momentum $p^{\prime}$, while the Compton wavelength $\lambda_{\mathrm{C}}$ is based on its rest mass $m_{0}$ - it is the wavelength of a photon whose energy is the same as the rest-mass energy of the particle. Thus, the de Broglie wavelength of a particle at rest is infinite, not the Compton wavelength. Highly non-relativistic particles can show wave properties over scales much larger than their Compton wavelength. A better way to think of the comparison is that when the coordinate de Broglie wavelength $\lambda^{\prime}$ or proper wavelength $\widetilde{\lambda}$ becomes shorter than the Compton wavelength $\lambda_{\mathrm{C}}$, relativistic effects become very important.

Defining $\lambda^{\prime} / \lambda_{\mathrm{C}}=\alpha$, we can rewrite

$$
N(r)=n(r)\left[1-\left(\frac{\alpha}{\Phi}\right)^{2}\right]^{1 / 2} .
$$

To preserve a real $N(r)$, we should maintain the condition that

$$
\alpha \leq \Phi(r) \forall r
$$

Equality implies that $N(r)=0$, which in turn suggests that $v_{\text {phase }}^{\prime}=v_{\text {group }}^{\prime}=$ $\infty$, an unphysical result violating special relativity. Therefore, $\alpha<\Phi(r)$. Likewise, for $\widetilde{N}(r)$ in Eq.(81), we must maintain $\widetilde{\lambda}<\lambda_{\mathrm{C}}$.

\section{Applications}

(i) Schwarzschild black hole

In this case, $r_{\text {th }}$ should be replaced by $r_{\text {hor }}=M / 2$. For ingoing photons, the indices are $n_{\mathrm{Sch}}(r)=\frac{\left(1+\frac{M}{2 r}\right)^{3}}{\left(1-\frac{M}{2 r}\right)}$ and $\widetilde{n}_{\mathrm{Sch}}(r)=\frac{1+\frac{M}{2 r}}{1-\frac{M}{2 r}}$ such that $n_{\mathrm{Sch}}(r)$, $\widetilde{n}_{\text {Sch }}(r) \rightarrow \infty$ at the horizon. Hence, the coefficients at the horizon as would appear to different observers are: $R_{\text {photon }}^{\text {a.o. }}=\widetilde{R}_{\text {photon }}^{\text {l.o. }}=1, T_{\text {photon }}^{\text {a.o. }}=\widetilde{T}_{\text {photon }}^{\text {l.o. }}=0$. The reflexivity is unity, hence the horizon is a stable surface, which is also 
independently known to be true. But if such reflection back into space really takes place, a black hole will not be a black hole any more!

This however would not happen because the time $t$ needed for the pulse to emerge from $r_{\text {hor }}$ and reach the asymptotic observer is infinite as the integrations $t=\int_{r_{\mathrm{hor}}}^{\infty} \frac{n_{\mathrm{Sch}}(r)}{c_{0}} d r$ and $\widetilde{t}=\int_{r_{\mathrm{hor}}}^{\infty} \frac{\widetilde{n}_{\mathrm{Sch}}(r)}{c_{0}} d r$ show. Light from the horizon will never reach the asymptotic observer justifying the name black hole. Therefore, there will be no true reflection in the ordinary sense - it has to be understood only by complementarity in the sense that the transmittivity coefficient is zero making up $R+T=1$, that is, light cannot transmit across the horizon either, so that the surface $r_{\text {hor }}=M / 2$ remains stable 7 . In the free space, $r \rightarrow \infty$, we have $n_{\mathrm{Sch}}(r)=1$ and so $R_{\text {photon }}^{\text {a.o. }}=0$ and $T_{\text {photon }}^{\text {a.o. }}=1$, that is, photons are fully transmitted, as expected.

For ingoing de Broglie waves with asymptotic speed $V$, the indices, using Eqs.(75) and (77), are

$$
\bar{N}_{\mathrm{Sch}}(r)=\frac{n_{\mathrm{Sch}}(r)}{\beta}\left[1-\alpha^{2}\left(1+\frac{M}{2 r}\right)^{4}\right]^{1 / 2} .
$$

Here again, using Eqs.(75),(77) and (81), we get $\bar{N}_{\mathrm{Sch}}(r), \widehat{N}_{\mathrm{Sch}}(r) \rightarrow \infty$ at the horizon, since $n_{\mathrm{Sch}}(r), \widetilde{n}_{\mathrm{Sch}}(r) \rightarrow \infty$ there. Hence, it follows that $\bar{R}_{\mathrm{dB}}^{\text {a.o. }}=$ $\widehat{R}_{\mathrm{dB}}^{\mathrm{loo}}=1, \bar{T}_{\mathrm{dB}}^{\mathrm{a} . \mathrm{o}}=\widehat{T}_{\mathrm{dB}}^{\mathrm{loo}}=0$, independently of the value of $V$ or the location of observers. However, even though the reflexivity is unity, time for the reflected de Broglie waves to reach asymptotic observer is infinite as $\bar{N}_{\text {Sch }}(r) \rightarrow \infty$. Hence, like in the case of light motion discussed above, since $\bar{t}=\int_{r_{\mathrm{hor}}}^{\infty} \frac{\bar{N}_{\mathrm{Sch}}(r)}{c_{0}} d r$ and $\widehat{t}=\int_{r_{\mathrm{hor}}}^{\infty} \frac{\widehat{N}_{\mathrm{Sch}}(r)}{c_{0}} d r$ is infinite, there will be no true reflection of matter

\footnotetext{
${ }^{7}$ No reflection, no transmission, where does all the light then go? For the Schwarzschild black hole of mass $M, n_{\mathrm{Sch}} \rightarrow \infty$ and $c^{\prime}=0$, as the horizon $r_{\text {hor }}=M / 2$ is approached by incoming light from infinity. Asymptotic observers thus see light standing "still" at $r_{\text {hor }}$, which is consistent with $T_{\text {photon }}^{\text {a.o. }}=0$ occurring exactly at $r_{\text {hor }}$. Similarly, local observers situated very near $r_{\text {hor }}$, on the other hand, measure proper quantities, $\widetilde{n}_{\text {Sch }} \rightarrow \infty$, again yielding $\widetilde{T}_{\text {photon }}^{\text {loo }}=0$. These observers would see light or particles never transmitting across the horizon but moving (as opposed to standing still) at the speed of light, $\widetilde{c}(\mathbf{r})=\left|\frac{d L}{d \tau}\right|=$ $c_{0}$, right on the horizon making it a "light sphere" (see, e.g., Ref.[39]) in conformity with general relativity. To either type of observers, asymptotic or local, complete lack of transmission across horizon is the reason for stability. We thus have an alternative description of the stable horizon, usually discussed within the perturbation scheme to the full spacetime fabric. The effective medium approach has no conflict with the usual geometric approach of general relativity, as also exemplified in Sec.4.
} 
particles and stability of the horizon is to be understood again only by the total lack of transmittivity across it.

In free space, at $r \rightarrow \infty$, using Eqs.(74) and (77), we get $\bar{N}_{\mathrm{Sch}}(\infty)=$ $\frac{N_{\infty}}{\beta}=1$. Using Eqs.(78) and (79), we then find $\bar{R}_{\mathrm{dB}}^{\text {a.o. }}=0$ and $\bar{T}_{\mathrm{dB}}^{\text {a.o. }}=1$, implying complete transmission of de Broglie waves into the medium, as expected. Hence, the Schwarzschild horizon is always a stable surface, no matter whether the perturbing pulse is a photon or a de Broglie wave.

(ii) Massive Ellis wormhole

The effective indices $n(r)$ and $N(r)$ are given in Eqs.(60) and (75) respectively and the isotropic throat radius is given by $r_{\mathrm{th}}=\frac{M}{2 \gamma}\left[\gamma+\sqrt{1+\gamma^{2}}\right]$ with $\alpha<\Phi\left(r_{\text {th }}\right)$ as required for the reality of $N(r)$. It is seen that, as $\gamma \rightarrow \infty$, $r_{\mathrm{th}} \rightarrow M$. This is the minimum of $r_{\mathrm{th}}$, while in general, $r_{\mathrm{th}}>M$ for finite $\gamma>0$. Also, Fig.1 shows $\Phi\left(r_{\text {th }}\right)$ vs $r_{\text {th }}$ for different $\gamma$ so that the required constraint $\alpha<\Phi\left(r_{\text {th }}\right)$ can be used, while choosing the value of $\alpha$. With these information at hand, choosing units such that $M=1$, and for a range of real non-zero values of $\gamma$, we graphically study the four pairs of coefficients.

For ingoing photons, Fig.2 displays the values of coefficients: The first thing to note is that the values of $(R, T)$ become nearly independent of the value of $\gamma$ for large $\gamma$ or at the minimum of throat $r_{\text {th }}$. The plot shows the perception of asymptotic observers, to whom the values appear to be $R_{\text {photon }}^{\text {a.o. }} \simeq 0.60$ and $T_{\text {photon }}^{\text {a.o. }} \simeq 0.40$ [Eqs. $\left.(64,65)\right]$ independently of the size of the throat. This means that for those observers there is a good probability that the ingoing photon will be reflected back from the throat providing chances of stability of the Ellis wormhole ${ }^{8}$. On the other hand, near-throat local observers observe the index as $\widetilde{n}$, which yields the coefficients $\widetilde{R}_{\text {photon }}^{\text {l.o. }} \simeq$ 0.20 and $\widetilde{T}_{\text {photon }}^{\text {loo. }} \simeq 0.80$ [Eqs. $\left.(66,67)\right]$ independently of the size of the throat. The probability of reflection is evidently very small meaning that the local observers are most likely to observe instability of the wormhole. The situation here is quite unlike the black hole horizon since at the throat, effective refractive indices $n(r)$ and $N(r)$ don't diverge.

The intriguing thing is that the probabilities and times of observations could conspire in such a way that, while the near-throat local observer finds low probability of reflection $\left(\widetilde{R}_{\text {photon }}^{\text {l.o. }} \simeq 0.20\right)$, meaning collapse (instability) of the wormhole, the asymptotic observer finds higher probability of reflection $\left(R_{\text {photon }}^{\text {a.o. }} \simeq 0.60\right)$, meaning that he is more likely to find the wormhole living

\footnotetext{
${ }^{8}$ Ordinary reflection is possible here because a wormhole throat is not a black hole horizon.
} 
(stability). This gives rise to a situation very similar to, but not exactly the same as, that of a ghost star. Since the Ellis wormhole spacetime including the throat surface is regular, the information of life prior to collapse would travel to asymptotic observers, who would then see it as a living wormhole consistent with higher probabilities for reflection observed by them. One could say in this case that the asymptotic observer is seeing a ghost wormhole (nothing to do with the ghost scalar field, $\varepsilon=-1$ ).

For ingoing de Broglie waves as perceived by asymptotic observers, we choose as an example, $\alpha=0.01<\Phi\left(r_{\text {th }}\right)$ and $\beta=0.2$. Fig.3. shows that $\bar{R}_{\mathrm{dB}}^{\text {a.o. }}$ reaches as high a value as 0.76 , while $\bar{T}_{\mathrm{dB}}^{\text {a.o. }}$ reaches a value 0.24 independently of the value of $\gamma$ or the size of the throat. In fact, as $\beta \rightarrow 0$, it follows that $\bar{N} \rightarrow \infty$, so that $\bar{R}_{\mathrm{dB}}^{\text {a.o. }} \rightarrow 1, \bar{T}_{\mathrm{dB}}^{\text {a.o. }} \rightarrow 0$, leading to total reflection, hence stability, in this limit. The same figure also shows that the higher the value of $\beta$, say 0.5 , the lower is the probability of reflection $\bar{R}_{\mathrm{dB}}^{\text {a.o. }}$, that is, the matter particles are more likely to penetrate the throat causing instability. This is a physically acceptable result.

Fig.4. shows the coefficients for ingoing de Broglie waves in the Ellis wormhole as perceived by the near-throat local observers. Plot shows de Broglie waves of high and low velocities $\beta=0.9$ and 0.01 . The lesser the velocity, the higher is the $\widehat{R}_{\mathrm{dB}}^{\text {lo. }}$. When $\beta \rightarrow 0$ (low velocity perturbation at asymptotics), stability is achieved: $\widehat{N} \rightarrow \infty, \widehat{R}_{\mathrm{dB}}^{\text {l.o. }} \rightarrow 1, \widehat{T}_{\mathrm{dB}}^{\text {l.o. }} \rightarrow 0$. However, stability of the wormhole is still not a certainty except in the limit $\beta \rightarrow 0$, because $R_{\text {photon }}^{\text {a.o. }}, \bar{R}_{\mathrm{dB}}^{\text {a.o. }}$ or $\widehat{R}_{\mathrm{dB}}^{\text {l.o. etc }}$ are not exactly unity, unlike in the case of Schwarzschild horizon. By the same token, instability is not a certainty either, when statistical arguments are used.

(iii) Massless Ellis wormhole

This is the most discussed wormhole, which is often used also as a good exemplar in the literature [40]. This is obtained from Eq.(47) by setting the Keplerian mass $M=m \gamma=0$. This value can be attained by two variants - either by setting $m=0, \gamma \neq 0$, which leads to a trivial flat spacetime, or by setting $m \neq 0, \gamma=0$, which leads to what one might call a zero (Keplerian) mass Ellis wormhole. The two mouths nonetheless have nonzero ADM masses $+m / 2$ and $-m / 2$ that add to zero. These masses are formed entirely by the scalar field $\phi(r)=2 \sqrt{2} \tan ^{-1}\left(\frac{2 r}{m}\right)$, as the integration of $\phi$ on two sides will show, and are responsible for gravitational microlensing, as worked out in detail by Abe [41].

For ingoing photon, we first note that, using Eq.(60) for $n(r)$, putting in 
it $M=m \gamma$, and choosing $\gamma=0$ in the resulting expression, we find

$$
n(r)=1+\frac{m^{2}}{4 r^{2}},
$$

and the throat, under similar choice, occur at a radius

$$
r_{\mathrm{th}}=\frac{m}{2}\left[\gamma+\sqrt{1+\gamma^{2}}\right] \rightarrow \frac{m}{2} .
$$

This gives, at the throat, $n\left(r_{\text {th }}\right)=2$ and for asymptotic observers, from Eqs. $(64,65)$, the exact values of the coefiicients

$$
R_{\text {photon }}^{\text {a.o. }}=\frac{1}{9}, T_{\text {photon }}^{\text {a.o. }}=\frac{8}{9} .
$$

An interesting result is that the near-throat local observers will notice $\widetilde{n}(r)=$ $n \Phi=1$ (since $n=\Phi^{-1}$ ), and consequently, from Eqs. $(66,67)$, we get the exact values

$$
\widetilde{R}_{\text {photon }}^{\text {l.o. }}=0, \widetilde{T}_{\text {photon }}^{\text {l.o. }}=1,
$$

which means that the photon will mimic its motion like in free space and completely transmit across the throat. The wormhole will appear definitively unstable to the local observers. Eqs.(90) and (91) together lead to a genuine possibility of zero mass ghost wormhole. This makes sense as Abe [41] has worked out lensing signatures of the living wormhole. Since the Keplerian mass $M=0$, its collapse might just mean its rapid expansion, that is expansion of the pure scalar field it is made of.

For ingoing matter waves, as before, using Eq.(54) and (75), we get the index

$$
\bar{N}(r)=\frac{n(r)}{\beta} \sqrt{1-\alpha^{2}\left(1+\frac{m^{2}}{4 r^{2}}\right)^{2}},
$$

which, at the throat $r=r_{\text {th }}=\frac{m}{2}$, reduces to

$$
\bar{N}\left(r_{\mathrm{th}}\right)=\frac{2}{\beta} \sqrt{1-4 \alpha^{2}} .
$$

The values of $\alpha<\frac{1}{2}$ and $0<\beta<1$ can be adjusted so that $0<\bar{N}\left(r_{\text {th }}\right)<\infty$. Thus, for $\beta \rightarrow 0, \alpha<\frac{1}{2}$, it is possible to make $\bar{N}\left(r_{\text {th }}\right) \rightarrow \infty$, so that one has $\bar{R}_{\mathrm{dB}}^{\text {a.o. }} \rightarrow 1, \bar{T}_{\mathrm{dB}}^{\text {a.o. }} \rightarrow 0$. This implies that the asymptotic observers will observe stability due to low asymptotic velocity of ingoing perturbation. For 
intermediate values of $\bar{N}\left(r_{\mathrm{th}}\right)$, the probabilities will change accordingly, and the arguments remain similar to the preceding ones. Finally, the near-throat local observers will perceive the index to be, from Eq.(81),

$$
\widehat{N}(r)=\frac{\widetilde{N}(r)}{\beta}=\frac{1}{\beta}\left[1-\left(\frac{\tilde{\lambda}}{\lambda_{\mathrm{C}}}\right)^{2}\right]^{1 / 2}
$$

which implies that $0<\widehat{N}(r)<\infty$. Thus, exactly as above, at the limit $\beta \rightarrow$ 0 , it follows that $\widehat{R}_{\mathrm{dB}}^{\text {l.o. }} \rightarrow 1, \widehat{T}_{\mathrm{dB}}^{\text {l.o. }} \rightarrow 0$, and the same arguments just as above apply. Local observers will observe stability, while asymptotic observers may or may not.

\section{Phantom wormhole}

Recently, Lobo, Parsaei and Riazi [3] have derived a number of phantom wormhole solutions. See also $[42,43]$ for a class of earlier solutions, which have been shown to be stable under perturbations within the class [44]. We shall here consider only the solution with bounded mass function [3] for exemplifying the probabilistic coefficients. Throughout the foregoing, we had used $r$ as the isotropic radial coordinate. The bounded mass function phantom wormhole they derived is in "standard" coordinates and is given by

$$
d s^{2}=\left(1+\frac{a R_{0}}{R}\right)^{1-\frac{1}{a}} c_{0}^{2} d t^{2}-\frac{d R^{2}}{1-\frac{R_{0}}{R}\left[\frac{a R_{0}}{R}+1-a\right]}-R^{2}\left(d \theta^{2}+\sin ^{2} \theta d \varphi^{2}\right)
$$

where $-1<a<0$, and $R$ is the standard radial coordinate. The throat appears at $R_{\mathrm{th}}=R_{0}$. The mass function is

$$
\mathcal{M}(R)=\frac{a R_{0}}{2}\left(\frac{R_{0}}{R}-1\right)
$$

The physical interpretation of the parameter $a$ is that it affects the redshifts of the signals originating from the throat or its nearby regions as follows

$$
z=\frac{\delta \lambda}{\lambda}=1-(1+a)^{\frac{1-a}{2 a}}
$$

We convert the solution to isotropic form by the radial transform $R \rightarrow r$ ,

$$
r=\left(\sqrt{R-R_{0}}+\sqrt{R+a R_{0}}\right)^{2} .
$$


The $r$-coordinate radius of the throat is

$$
r_{\text {th }}=(1+a) R_{0}
$$

and inverting Eq.(98), we find

$$
R(r)=\frac{(1+a) r^{4}-2(a-1) r^{2} r_{\mathrm{th}}+(1+a) r_{\mathrm{th}}^{2}}{4(1+a) r^{2}} .
$$

The metric (95) can be re-written in the isotropic form

$$
\begin{gathered}
d s^{2}=\left[1+\frac{a R_{0}}{R(r)}\right]^{1-\frac{1}{a}} c_{0}^{2} d t^{2}-\mu^{2}[R(r)]\left[d r^{2}+r^{2}\left(d \theta^{2}+\sin ^{2} \theta d \varphi^{2}\right)\right] \\
\mu(R)=\Phi^{-1}=\frac{R}{\left(\sqrt{R-R_{0}}+\sqrt{R+a R_{0}}\right)^{2}}
\end{gathered}
$$

where $R$ is given by Eq.(100). Therefore, the refractive index, as perceived by asymptotic observers, is

$$
n[R(r)]=\Phi^{-1} \Omega^{-1}=\mu(R)\left[1+\frac{a R_{0}}{R}\right]^{\frac{1}{2}\left(\frac{1}{a}-1\right)}
$$

and similarly, the index as perceived by near-throat local observers is

$$
\widetilde{n}[R(r)]=n \Phi=\left[1+\frac{a R_{0}}{R}\right]^{\frac{1}{2}\left(\frac{1}{a}-1\right)}
$$

At the throat $R_{\mathrm{th}}=R_{0}$, the indices have the values

$$
n\left(R_{\mathrm{th}}\right)=(1+a)^{\frac{1}{2 a}-\frac{3}{2}}, \widetilde{n}\left(R_{\mathrm{th}}\right)=(1+a)^{\frac{1}{2 a}-\frac{1}{2}} .
$$

Thus, as $a \rightarrow-1$, we have $n\left(R_{\mathrm{th}}\right), \widetilde{n}\left(R_{\mathrm{th}}\right) \rightarrow \infty$, so that $\bar{N}\left(R_{\mathrm{th}}\right), \widehat{N}\left(R_{\mathrm{th}}\right) \rightarrow$ $\infty$ irrespective of the value of $0<\beta<1$, finally leading to

$$
\begin{aligned}
R_{\text {photon }}^{\text {a.o. }} & =\widetilde{R}_{\text {photon }}^{\text {l.o. }}=1, T_{\text {photon }}^{\text {a.o. }}=\widetilde{T}_{\text {photon }}^{\text {l.o. }}=0, \\
\bar{R}_{\mathrm{dB}}^{\text {a.o. }} & =\widehat{R}_{\mathrm{dB}}^{\text {l.o. }}=1, \bar{T}_{\mathrm{dB}}^{\text {a.o. }}=\widehat{T}_{\mathrm{dB}}^{\text {l.o. }}=0 .
\end{aligned}
$$

and, as $a \rightarrow 0$, we have $n\left(R_{\mathrm{th}}\right), \widetilde{n}\left(R_{\mathrm{th}}\right) \rightarrow \sqrt{e}$, so that likewise

$$
\begin{aligned}
R_{\text {photon }}^{\text {a.o. }} & =\widetilde{R}_{\text {photon }}^{\text {l.o. }}=0.06, T_{\text {photon }}^{\text {a.o. }}=\widetilde{T}_{\text {photon }}^{\text {loo. }}=0.94 \\
\bar{R}_{\mathrm{dB}}^{\text {a.o. }} & =\widehat{R}_{\mathrm{dB}}^{\text {l.o. }}=0.06, \bar{T}_{\mathrm{dB}}^{\text {a.o. }}=\widehat{T}_{\mathrm{dB}}^{\text {l.o. }}=0.94 .
\end{aligned}
$$


It is quite evident from the metric (95) itself that, in the extreme limit $a \rightarrow-1$, the throat behaves like the Schwarzschild horizon, where $\Omega^{2} \rightarrow 0$. Consistent with such behavior, the coefficients in Eqs. $(105,106)$ indicate that, to asymptotic and local observers, the phantom wormhole will appear almost definitely stable no matter whether the perturbing pulse is an ingoing photon or a matter particle. On the contrary, in the other extreme limit, $a \rightarrow 0$, all observers will find the wormhole to be almost definitely unstable, as the coefficients in Eqs. $(107,108)$ show. For intermediate values of $a$, the asymptotic and local observers would see all combinations of coefficients leading to $R+T=1$, so again there are possibilities that the asymptotic observer will see ghost phantom wormholes.

The indeterminacy a la Tangherlini leading to wormhole (in)stability might seem somewhat counter-intuitive at first sight, but would make sense if such indeterminacy could be translated into observable deterministic signatures of ghost wormholes. However, in the absence of known experiments on ghost wormholes, we propose below a possible thought experiment, where we explicitly calculate those signatures.

\section{A thought experiment}

The experiment exploits, for instance, different gravitational lensing properties of black and wormholes (for some earlier works on such properties, see [45-48]). For simplicity, we assume that there is only one object in the Universe and that local $\left(\widetilde{O}_{\text {l.o }}\right)$ and asymptotic observers $\left(O_{\text {a.o. }}\right)$ exchange information by means of some signal about their lensing measurements of the object. Further, we assume that they know the works by González et al. $[5,6]$ about Ellis wormhole's deterministic collapse into Schwarzschild black hole corresponding to $(R, T)=(0,1)$. On the other hand, as shown above, the coefficients need not always be exact certainties like $(R, T)=(0,1)$ or $(1,0)$. Hence, both observers can have fractional probabilities, however big or small, of observing (in)stabilities of the throat leading to black or wormholes, as the case may be. Both objects can act as lens $(L)$ giving images of background sources $(S)$ that are observed by observers $(O)$. When $L, S$ and $O$ are aligned along a line, the images form a ring called the Einstein ring centered at the lens; otherwise there will be discrete images on both sides of the optic axis $O L$ at angular locations denoted by $\theta$.

Coming to lensing observations, an essential constraint is required to be fulfilled: The light coming from the background source to the observer should not get wound up right on the photon sphere centered at the lens, with the 
sphere radius $r=r_{\mathrm{ps}}$ defined by the largest root of the equation

$$
\frac{1}{A} \frac{d A}{d r}=\frac{1}{C} \frac{d C}{d r},
$$

for a generic metric

$$
d \tau^{2}=A(r) d t^{2}-B(r) d r^{2}-C(r)\left(d \theta^{2}+\sin ^{2} \theta d \varphi^{2}\right) .
$$

If the incoming light is eternally captured by the photon sphere, no image will be formed.

However, the incoming light rays that pass very near to the photon sphere yield strong field lensing observables [45] measured by both the observers $\left(\widetilde{O}_{\text {l.o }}\right)$ and $\left(O_{\text {a.o. }}\right)$. The easiest observable to evaluate is the minimum impact parameter $u_{m}$, and this information alone can already distinguish between Schwarzschild and other types of geometry such as wormholes. Therefore, we consider situations that guarantee $u_{m}>r_{\mathrm{ps}}$. The parameter $u_{m}$ is proportional to the separation $\theta_{\infty}$ between each set of relativistic images with respect to the central lens given by [45]

$$
u_{m}=D_{\mathrm{OL}} \theta_{\infty},
$$

where $D_{\mathrm{OL}}$ is the distance between the observer $(O)$ and the lens $(L)$ and

$$
u_{m}\left(r_{\mathrm{ps}}\right)=\sqrt{C\left(r_{\mathrm{ps}}\right) / A\left(r_{\mathrm{ps}}\right)} .
$$

The radius of the photon sphere for the Ellis wormhole metric (52-55) is

$$
r_{\mathrm{ps}}=M\left(1+\frac{1}{2} \sqrt{4+\frac{1}{\gamma^{2}}}\right) .
$$

Evidently, it is the evaluation of $u_{m}$, or equivalently $\theta_{\infty}$, defined by the metric functions $C(r), A(r)$ that distinguishes one spacetime from the other.

Note that, for fixed $M$, it is only the value of $\gamma$ that determines the refractive indices $(n, \widetilde{n})$, hence the probabilistic coefficients $(R, T)$ or $(\widetilde{R}, \widetilde{T})$ at the throat, observed by local and asymptotic observers. These probabilistic coefficients in turn dictate which observer is likely to observe what $\theta_{\infty}$. Thus, all deterministic observations boil down to measuring the values of $\gamma$, as it were, by truly measuring $\theta_{\infty}$. In Sec.7(ii), the dependence on $\gamma$ was already 
demonstrated but the situation was idealized $(M=1)$ without reference to any practical (lensing) measurement.

We now consider a practical situation: It is widely believed that the center of our galaxy hosts a supermassive black hole (BH) with mass $M=$ $2.8 \times 10^{6} M_{\odot}[45-50]$. Taking $D_{\mathrm{OL}}=8.5 \mathrm{kpc}=2.62 \times 10^{22} \mathrm{~cm}$ as the distance between the local observer $\left(\widetilde{O}_{1.0}\right)$ identified at the sun and the lens $(L)$ at the center of our galaxy, Virbhadra and Ellis [51] found that $\theta_{\infty}^{\mathrm{BH}} \sim 17$ microarcsecs. In principle, such a resolution at the microarcsec level is reachable by actual VLBI projects in the near future [45].

Now we recall the calculations of Sec.5, where we showed that only the exclusive value $\gamma=-i$ yielded a Schwarzschild black hole. Using this imaginary $\gamma$, the values $r_{\mathrm{ps}}=7.73 \times 10^{11} \mathrm{~cm}, u_{m}=2.15 \times 10^{12} \mathrm{~cm}$ and $\theta_{\infty}^{\mathrm{BH}}=16.943$ microarcsecs can be accurately calculated from Eqs. (111-113), once the independently observed values of $M$ and $D_{\mathrm{OL}}$ are put in. We also notice that the condition $u_{m}>r_{\mathrm{ps}}$ is fulfilled. If measurements confirm this value of $\theta_{\infty}$ within experimental errors, then $\widetilde{O}_{1 . o}$ concludes a Schwarzschild black hole. The precision must be very high in order to arrive at this conclusion. Even a small deviation from this value of $\theta_{\infty}^{\mathrm{BH}}=16.943$ microarcsecs would mean that we are perhaps looking at the positive mass mouth $M$ of the Ellis wormhole at the galactic center instead of a black hole. For instance, the measurements of $\widetilde{O}_{\text {l.o }}$ might correspond to a real $\gamma$ (wormhole), say, $\gamma=50$. With the other observed data $M=2.8 \times 10^{6} M_{\odot}$ and $D_{\mathrm{OL}}=8.5 \mathrm{kpc}$ remaining the same, he would then measure the wormhole $(\mathrm{WH})$ value $\theta_{\infty}^{\mathrm{WH}}=17.736$ microarcsecs, not too different from the black hole value $\theta_{\infty}^{\mathrm{BH}}$. The VLBI missions should have a very high precision to be able to distinguish such small differences $\left(\leq 0.793\right.$ microarcsec). Note that $\theta_{\infty}^{\mathrm{WH}}$ saturates to the value above as $\gamma \rightarrow \infty$ and so cannot be reduced further.

To find the observations of the asymptotic observer $\left(O_{\text {a.o }}\right)$, we really do not go to $r=\infty$, but just consider him to be located far away from $\left(\widetilde{O}_{\mathrm{l} . \mathrm{o}}\right)$, e.g., $D_{\mathrm{OL}}=\kappa r_{\mathrm{ps}}>8.5 \mathrm{kpc}$, where $\kappa=3 \times 10^{10}$ (say). Assuming the scalar field contribution to be small, say $\gamma=0.1$ (wormhole) at this large distance from the lens, we find the following values: The photon sphere of the wormhole has a radius $r_{\mathrm{ps}}=2.52 \times 10^{12} \mathrm{~cm}$, observer-lens distance $D_{\mathrm{OL}}=\kappa r_{\mathrm{ps}}=24.57 \mathrm{kpc}=7.58 \times 10^{22} \mathrm{~cm}$ and $u_{m}=5.56 \times 10^{12} \mathrm{~cm}$, which are of the same order of magnitude as, but about three times larger than, the Schwarzschild values. Also the condition $u_{m}>r_{\mathrm{ps}}$ is fulfilled. With the same value $M=2.8 \times 10^{6} M_{\odot}$, Eq.(111) now yields $\theta_{\infty}^{\mathrm{WH}}=15.135$ 
microarcsecs, which is slightly lower than that of the Schwarzschild value. Measuring this value of $\theta_{\infty}^{\mathrm{WH}}$ (for that matter, any value differing from the reference Schwarzschild value $\theta_{\infty}^{\mathrm{BH}}=16.943$ microarcsecs), $O_{\text {a.o }}$ will conclude a wormhole (since $\gamma$ is now real). By receiving the Schwarzschild black hole data from $\widetilde{O}_{\mathrm{l} . \mathrm{o}}$, the distant observer $O_{\text {a.o }}$ further concludes that the wormhole he sees must be a ghost since, by assumption, there is only one object and that both observers know that Schwarzschild black hole is only a result of Ellis wormhole's deterministic collapse $[5,6,7]$.

The observer $O_{\text {a.o }}$ also has the possibility to observe his black hole value of $\theta_{\infty}$, for which $\gamma=-i$. Then, for the same mass $M=2.8 \times 10^{6} M_{\odot}$ but distance $D_{\mathrm{OL}}=24.57 \mathrm{kpc}$, he should measure $\theta_{\infty}^{\mathrm{BH}}=5.859$ microarcsecs. Thus, if $O_{\text {a.o }}$ has to observe a black hole, the accuracy of measurement has to be extremely high, unreachable in the near future, but cannot be ruled out in principle. All the above arguments show that the converse can also happen, that is $\widetilde{O}_{1.0}$ can observe a wormhole value $\theta_{\infty}^{\mathrm{WH}}=17.736$ microarcsecs, as already discussed above, while $O_{\text {a.o }}$ can observe a black hole measuring $\theta_{\infty}^{\mathrm{BH}}=5.859$ microarcsecs. One could then say by reversing the previous arguments that it is $\widetilde{O}_{1.0}$ that sees a ghost wormhole. No ghost phenomenon appears, when both the observers together measure either real or imaginary value of $\gamma$, that is, when their conclusions are in agreement. However, there is no possibility of ghost black holes as further collapse of a black hole into anything else is not known.

\section{Conclusions}

It has been concluded in Refs.[5,6,7] on the grounds of metric gravity master equation that the Ellis wormhole is unstable to linear and non-linear perturbations. While their deterministic conclusion is correct, we have revisited in this paper the issue of stability from the viewpoint of local and asympotic observers applying the results of Tangherlini's non-deterministic statistical framework dealing with photon motion in real optical medium. We have extended the application to include also the motion of matter particles. Tangherlini's approach is essentially heuristic with a pre-quantum flavor but, interestingly, the expressions of coefficients [Eqs.(6)] exactly resemble the probabilities obtained by solving the Schrödinger equation involving a certain potential [36].

It is indeed remarkable that the optics-type equations, such as $p^{\prime}=n(r) p$ or $p^{\prime} \lambda^{\prime}=p \lambda=$ constant, are already non-trivially embedded in the formalism of general relativity expressed in the language of the medium analogue. 
Though the analogue is secondary to metric gravity, it is nonetheless quite useful in that it not only describes the known general relativistic kinematics in a familiar optical language, but also leads to new insights in gravity coming from experience in the true optical regime [10-18]. This success leads us to expect that the application of Tangherlini's formulation to effective medium could yield genuine predictions of reflection and transmission probabilities, and by implication, about wormhole (in)stability back in the general relativity regime.

The following are our new results:

(i) The expansion of the metric coefficients or the refractive index of the Ellis wormhole [see Eqs.(61),(62)] show that they can never trivially reduce to a Schwarzschild black hole for any real $\gamma$, unlike in the case of Ellis I solution (naked singularity). Consequently, Ellis wormhole would never seem to collapse to a Schwarzschild black hole, contrary to the "strong" indication stated in Ref.[6]. We show that this need not be the case. By Wick-like complex transformations, it is possible to show that the Ellis wormhole can indeed decay into a Schwarzschild black hole and that the throat would reduce to the horizon.

(ii) We show that the perception of stability of the Ellis wormhole depends on the location of the observer. When the pulse is an ingoing photon (Fig.2), to asymptotic observers, the coefficients appear to be $R_{\text {photon }}^{\text {a.o. }} \simeq 0.60$ and $T_{\text {photon }}^{\text {a.o. }} \simeq 0.40$ [Eqs. $\left.(64,65)\right]$ independently of the size of the throat. This means that those observers are more likely to see the ingoing photon reflected back from the throat providing chances of stability of the Ellis wormhole. On the other hand, near-throat local observers observe the index as $\tilde{n}$, which yields the coefficients $\widetilde{R}_{\text {photon }}^{\text {l.o. }} \simeq 0.20$ and $\widetilde{T}_{\text {photon }}^{\text {l.o. }} \simeq 0.80$, meaning that the local observers are most likely to observe transmission through the throat, hence instability of the wormhole. The two observations put together give rise to a situation leading to a ghost wormhole, very similar to a ghost star [Sec.7(ii)]. For massless Ellis wormhole, the coefficients have the set of values $\left(R_{\text {photon }}^{\text {a.o. }}=\frac{1}{9}, T_{\text {photon }}^{\text {a.o. }}=\frac{8}{9}\right)$ and $\left(\widetilde{R}_{\text {photon }}^{\text {l.o. }}=0, \widetilde{T}_{\text {photon }}^{\text {l.o. }}=1\right)$ at the throat. Hence, asymptotic observers cannot definitively conclude instability, whereas local observers can and there again emerges the possibility of a zero mass ghost wormhole.

(iii) We have extended the formalism to include de Broglie matter waves such that $p^{\prime} / p=\bar{N}$ and plotted the corresponding coefficients. It was shown that the coefficients in general depend not only on the location of the observer 
but also on the asymptotic velocity of the ingoing matter particle. However, for low velocity particles, meaning $\beta \rightarrow 0$, it follows that $\bar{R}_{\mathrm{dB}}^{\text {a.o. }}, \widehat{R}_{\mathrm{dB}}^{\text {l.o. }} \rightarrow 1$, implying stability. As such, for a low momentum impact to the throat, this stability is no surprise. Fig. 3 displays the variation of coefficients with the variation of $\beta$. It is seen that, even for $\beta=0.2$, which is not too small a velocity, the coefficient $\bar{R}_{\mathrm{dB}}^{\text {a.o. }}$ could reach as high a value as 0.76, [see Sec.7(ii)].

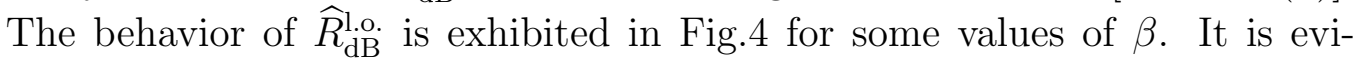
dent that local observers are also most likely to see the wormhole stable for moderate impact at the throat: The lower the $\beta$, the higher is the reflection probability and conversely.

(iv) Wormholes are supposed to have been born in the early universe due to high energetic processes and ought to have been inflated into macroscopic size today due to cosmic expansion driven by the phantom energy [52]. The phantom wormholes recently obtained by Lobo, Parsaei and Riazi [3] could thus be a real possibility, at least as real as the phantom energy itself. The question is: Did they survive the bombardment by photons and particles? Our analysis indicates that extreme phantom wormholes $(a \rightarrow-1)$ should have survived since the coefficients in Eqs. $(105,106)$ indicate that, to asymptotic and local observers, $R \rightarrow 1$ and the wormhole would appear almost definitely stable independently of whether the perturbing pulse is an ingoing photon or a matter particle (Fig.5). For intermediate values, $-1<a<0$, the asymptotic and local observers would see all possible combinations of coefficients leading to $R+T=1$, so again there are possibilities that the asymptotic observer will see ghost phantom wormholes.

The main conclusion is that, as long as the coefficients do not approach strict values $R=1, T=0$, as happens in the case of Schwarzschild black hole or the extreme phantom wormhole, there is always the possibility that, while near-throat local observers see instability (low probability of reflection), the asymptotic observers see stability (high probability of reflection), thus leading to ghost wormholes due to exchange of observational data between them, as explained in Sec.7(ii).

We wish to comment on some of the challenges: Though the ghost field $(\varepsilon=-1)$ or phantom energy have come to stay with us for various reasons, the exotic matter they bring along poses lots of challenges. One is of course the proven classical instability $[5-8,53]$ threatening the very survival of wormholes up to today. Mixed configurations such as neutron star-plus-wormhole systems [54] are also shown to be unstable to linear perturbations [55]. Ex- 
otic matter leads to serious problems at the quantum level too, where the negative kinetic term leads to the possibility that the energy density could become arbitrarily negative for high frequency oscillations [56]. However, the dilatonic Einstein-Gauss-Bonnet wormholes $[57,58]$ not threaded by exotic matter are shown to be stable. Coming to present work, Tangherlini's non-deterministic arguments seem to provide a different route to approach the question of stability of wormholes.

A major challenge is posed by a very pertinent question: How to understand the deterministic validity of the counter-intuitive non-deterministic results on the (in)stability of Ellis or phantom wormholes? $?^{9}$ This question arises mainly because the effective medium description is only an artificial analogue and not a ponderable real medium, so it's not clear how genuine are the results derived from such an analogue. On the other hand, such non-deterministic results must have deterministic counterpart for them to be observationally meaningful. To that end, we discussed in Sec.9, as unequivocally and convincingly as possible, exactly what numerical values are to be expected as deterministic counterparts of the indeterminacy under consideration. Therefore, we argue that the non-deterministic coefficients $(R, T)$ lurking in the analogue medium is at play throwing up genuine observable results on the (in)stability of wormholes in the gravity field.

A few cautionary notes seem to be in order: The non-deterministic results are not fait accompli but translate into observable new predictions yet to be tested by actual experiments. Next, the time scale associated with this instability is macroscopic as Tangherlini's approach is non-quantum, the Planck constant $\hbar$ cancelled out of the formulation [see (T3) of Sec.2]. The scale is of the order of the areal radius of the throat divided by the speed of light, which is of the order of a few microseconds for a throat of radius of the order of kilometers. Such time scales have to be factored in, while devising measurements. Then, we showed in Sec.4 that the Pound-Rebka frequency shift experiment in the Schwarzschild gravity field is nothing but the momentum increase of photon in the analogue medium. But this is purely a heuristic example, not to be taken literally. Since the analogue medium cannot be seen or touched like the real one, it is only our modest hope, and not a claim, that different values of observables as an effect of background indeterminism should nonetheless be measured in the gravity field - unless decisively ruled out by actual experiments.

\footnotetext{
${ }^{9}$ We thank an anonymous referee for raising this critical question.
} 
Such a hope is somewhat loosely grounded to a philosophical viewpoint: One could argue that the whole edifice of theoretical physics is built on mathematical models that work "unreasonably" well [59]. For example, Einstein's theory itself is an effective geometric model of gravity - abstract geometry that cannot be seen or touched but its observable predictions have been accurately tested giving the model the credibility it deserves. Similarly, our effective medium approach, though a secondary construct based on a priori knowledge of the Ellis wormhole geometry, would be physically relevant only if the predicted indeterminacy could be translated into observable deterministic signatures of ghost wormholes.

Since there are as yet no readymade experiments supporting or refuting such ghost phenomenon, we devised a possible thought experiment in Sec.9 and calculated the relevant lensing observable as an attempt to answer the question posed above. We provided arguments for the deterministic validity of (in)stability of Ellis/phantom wormholes, the observable signatures of (in)stability depending on the location of the observers and values of $\gamma$. Such location dependent observation is a hallmark of general relativity and is preserved also in our analysis: It was shown that the simplest strong lensing observable, the minimum impact parameter $u_{m}$ (or equivalently $\theta_{\infty}$ ), measured by observers stationed at different locations would be different. Thus, while a local observer measuring $\theta_{\infty}^{\mathrm{BH}}=16.943$ microarcsecs concludes a Schwarzschild black hole $(\gamma=-i$ strictly), another observer at a distant location measuring a different value of $\theta_{\infty}^{\mathrm{WH}}$ will conclude a wormhole $(\gamma$ real and arbitrary). By exchanging information between them, the latter observer can deterministically conclude that he is observing a ghost wormhole. The converse situation is also possible as explained in Sec.9.

Finally, we remark that the whole preceding development is a consequence of Tangherlini's ingenious idea of pre-quantum non-deterministic reflection and transmission coefficients, which are remarkably the same as those obtained from the quantum Schrödinger equation with an appropriate potential [36]. When a full consistent theory of quantum gravity will be available in the future, we hope that the concept of ghost wormhole might just manage to survive.

\section{Acknowledgments}

The authors are indebted to Guzel Kutdusova and Arunava Bhadra for useful conversations. Part of the paper was delivered by one of us (KKN) as an invited S.N. Bose Memorial Lecture on 07 Sept.2015 at the Calcutta Mathematical Society, India. 


\section{References}

[1] F.R. Tangherlini, Phys. Rev. A 12, 139 (1975).

[2] H.G. Ellis, J. Math. Phys. 14, 104 (1973); Errata: J. Math. Phys. 15, 520 (1974).

[3] F.S. N. Lobo, F. Parsaei and N. Riazi, Phys. Rev. D 87, 084030 (2013).

[4] K.A. Bronnikov, Acta Phys. Polon. B 4, 251 (1973).

[5] J.A. González, F.S. Guzmán and O. Sarbach, Class. Quant. Grav. 26, 015010 (2009).

[6] J.A. González, F.S. Guzmán and O. Sarbach, Class. Quant. Grav. 26, 015011 (2009).

[7] K.A. Bronnikov, J.C. Fabris and A. Zhidenko, Europhys J. C 71, 1791 (2011).

[8] K. A. Bronnikov, R. A. Konoplya and A. Zhidenko, Phys. Rev. D 86, 024028 (2012). See also: K.A. Bronnikov and S. Grinyok, Grav. Cosmol. 10, 237 (2004); K.A. Bronnikov and A.A. Starobinsky, JETP Lett. 85,1 (2007).

[9] C. Armendáriz-Picón, Phys. Rev. D 65, 104010 (2002).

[10] K.K. Nandi and A. Islam, Am. J. Phys. 63, 251 (1995).

[11] A.S. Eddington, Space, Time and Gravitation (Cambridge U.P., Cambridge, 1920, reprinted 1987).

[12] F. de Felice, Gen. Relat. Grav. 2, 347 (1971).

[13] J. Evans, K.K. Nandi and A. Islam, Am. J. Phys. 64, 1404 (1996).

[14] J. Evans, P.M. Alsing, S. Giorgetti and K.K. Nandi, Am. J. Phys. 69, 1103 (2001).

[15] P.M. Alsing, J.C. Evans and K.K. Nandi, Gen. Relat. Grav. 33, 1459 (2001).

[16] K.K. Nandi, Y.Z. Zhang, P.M. Alsing, J.C. Evans and A. Bhadra, Phys. Rev. D 67, 025002 (2003).

[17] J. Evans, K.K. Nandi and A. Islam, Gen. Relat. Grav. 28, 413 (1996).

[18] P.M. Alsing, Am. J. Phys. 66, 779 (1998).

[19] J. Javanainen and J. Ruostekoski, Phys. Rev. A 52, 3033 (1995).

[20] L.V. Hau, S.E. Harris, Z. Dutton and C.H. Behroozi, Nature (London) 397, 594 (1999).

[21] U. Leonhardt and P. Piwnicki, Phys. Rev. A 60, 4301 (1999).

[22] U. Leonhardt and P. Piwnicki, Phys. Rev. Lett. 84, 822 (2000); ibid. 85, 5253 (2000).

[23] M. Visser, Phys. Rev. Lett. 85, 5252 (2000). 
[24] U. R. Fischer and M. Visser, Phys. Rev. Lett. 88, 110201 (2002).

[25] Dutton,Z./VestergaardHau,L., Phys. Rev. A 70, 053831 (2004).

[26] W. G. Unruh, Phys. Rev. Lett. 46,1351(1981).

[27] W.G. Unruh, Phys. Rev. D 51, 2827 (1995).

[28] M. Visser, Class. Quant. Grav. 15, 1767 (1998).

[29] M. Visser, Phys. Rev. Lett. 80, 3436 (1998).

[30] S. Liberati, S. Sonego and M. Visser, Class. Quant. Grav. 17, 2903 (2000).

[31] M. Visser, C. Barcelo and S. Liberati, Gen. Rel. Grav. 34, 1719 (2002).

[32] J. H. Poynting, Philos. Mag. 9, 393 (1905).

[33] R. V. Jones, Nature (London), 167, 439 (1951).

[34] R. V. Jones and J. C. S. Richards, Proc. Roy. Soc. A 221, 480 (1954).

[35] A. Ashkin and J. M. Dziedzic, Phys. Rev. Lett. 30,139 (1973).

[36] D. Bohm, Quantum Theory (Prentice-Hall, Inc., New York, 1951), p. 235

[37] S.K. Bose, Introduction to General Relativity (Wiley Eastern, New Delhi, 1980).

[38] M.A. Scheel, S.L. Shapiro and S.A. Teukolsky, Phys. Rev. D 51, 4208 (1995); ibid. 51, 4236 (1995).

[39] W. Rindler, Essentials of Relativity (Springer-Verlag, New York, 1977), p.189

[40] M.S. Morris and K.S. Thorne, Am. J. Phys. 56, 395 (1988).

[41] F. Abe, Astrophys. J. 725, 787 (2010).

[42] S.V. Sushkov, Phys. Rev. D 71, 043520 (2005).

[43] F.S.N. Lobo, Phys. Rev. D 71, 084011 (2005).

[44] F.S.N. Lobo, Phys. Rev. D 71, 124022 (2005).

[45] V. Bozza, Phys. Rev. D 66, 103001 (2002).

[46] K.S. Virbhadra and G.F.R. Ellis, Phys. Rev. D 62, 084003 (2000).

[47] C. R. Keeton and A.O. Petters, Phys. Rev. D 72,104006 (2005);

ibid. D 73, 044024 (2006); ibid. D 73, 104032 (2006).

[48] K.K. Nandi, Y.Z. Zhang and A.V. Zakharov, Phys. Rev. D 74, 024020 (2006).

[49] J.B. Hartle, Gravity - An introduction to general relativity (Pearson Inc., San Francisco, 2003).

[50] D. Richstone et al., Nature (London), 395, A14 (1998).

[51] K.S. Virbhadra and G.F.R. Ellis, Phys. Rev. D 62, 084003 (2000). 
[52] S.V. Sushkov and S-W. Kim, Gen. Rel. Grav. 36,1671 (2004).

[53] H. -a. Shinkai and S. A. Hayward, Phys. Rev. D 66, 044005 (2002).

[54] V. Dzhunushaliev, V. Folomeev, B. Kleihaus and J. Kunz, Phys. Rev. D 85, 124028 (2012).

[55] V. Dzhunushaliev, V. Folomeev, B. Kleihaus and J. Kunz, Phys. Rev. D 87, 104036 (2013).

[56] S. V. Sushkov and Y.-Z. Zhang, Phys. Rev. D 77, 024042 (2008).

[57] P. Kanti, B. Kleihaus and J. Kunz, Phys. Rev. Lett. 107, 271101 (2011).

[58] P. Kanti, B. Kleihaus and J. Kunz, Phys. Rev. D 85, 044007 (2012).

[59] M. Steiner, The applicability of mathematics as a philosophical problem (Harvard University Press, Massachusetts, 1998).

\section{Figure Captions}




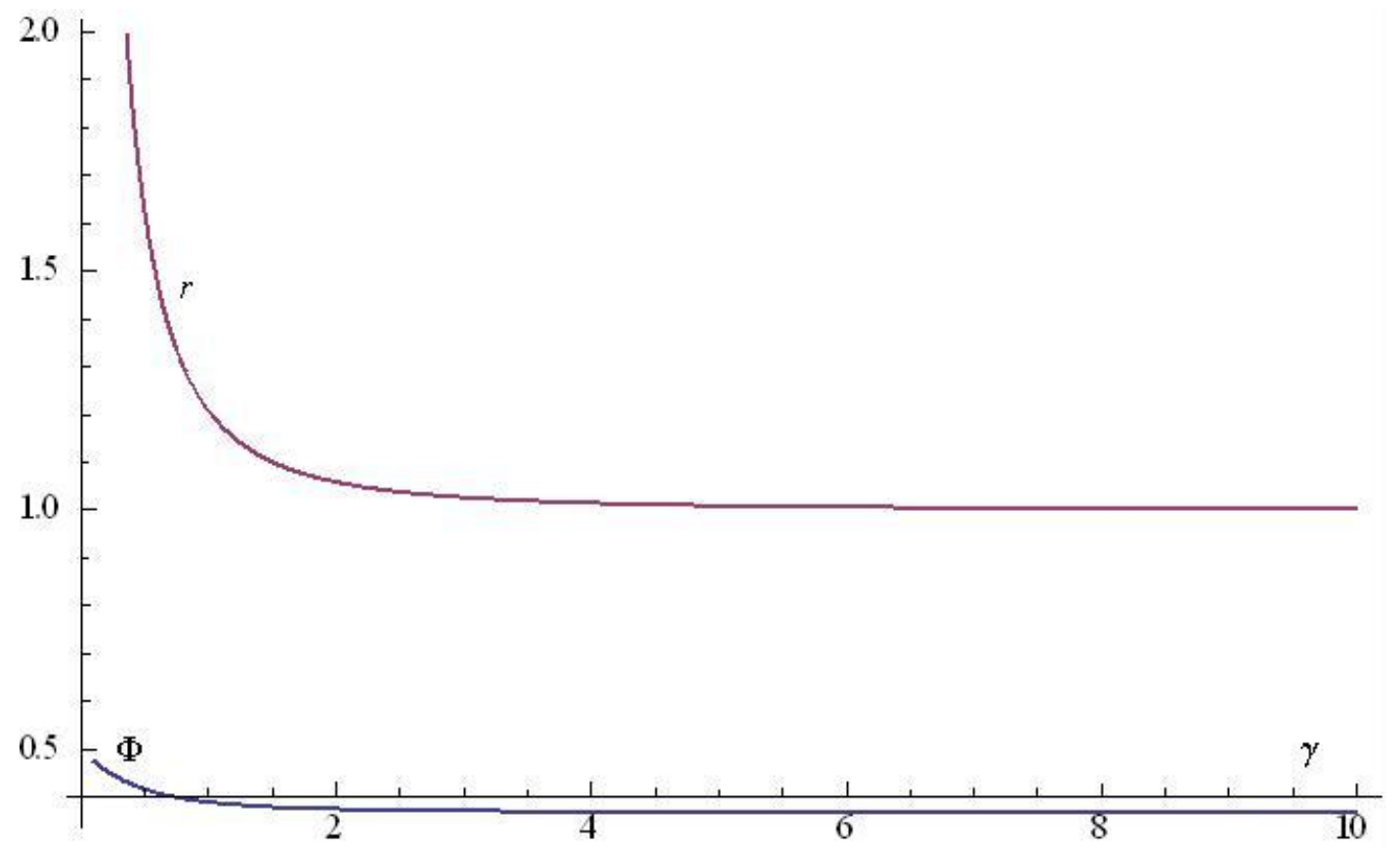

Figure 1: Plot of $\Phi\left(r_{\mathrm{th}}\right)$ and $r_{\mathrm{th}}(\gamma)$ vs $\gamma$. The larger the value of $\gamma$, the smaller the value of $r_{\mathrm{th}}$ and $\Phi\left(r_{\mathrm{th}}\right)$. One should choose $\alpha$ such that $\alpha<\Phi\left(r_{\mathrm{th}}\right)$ needed for the reality of $N(r)$. 


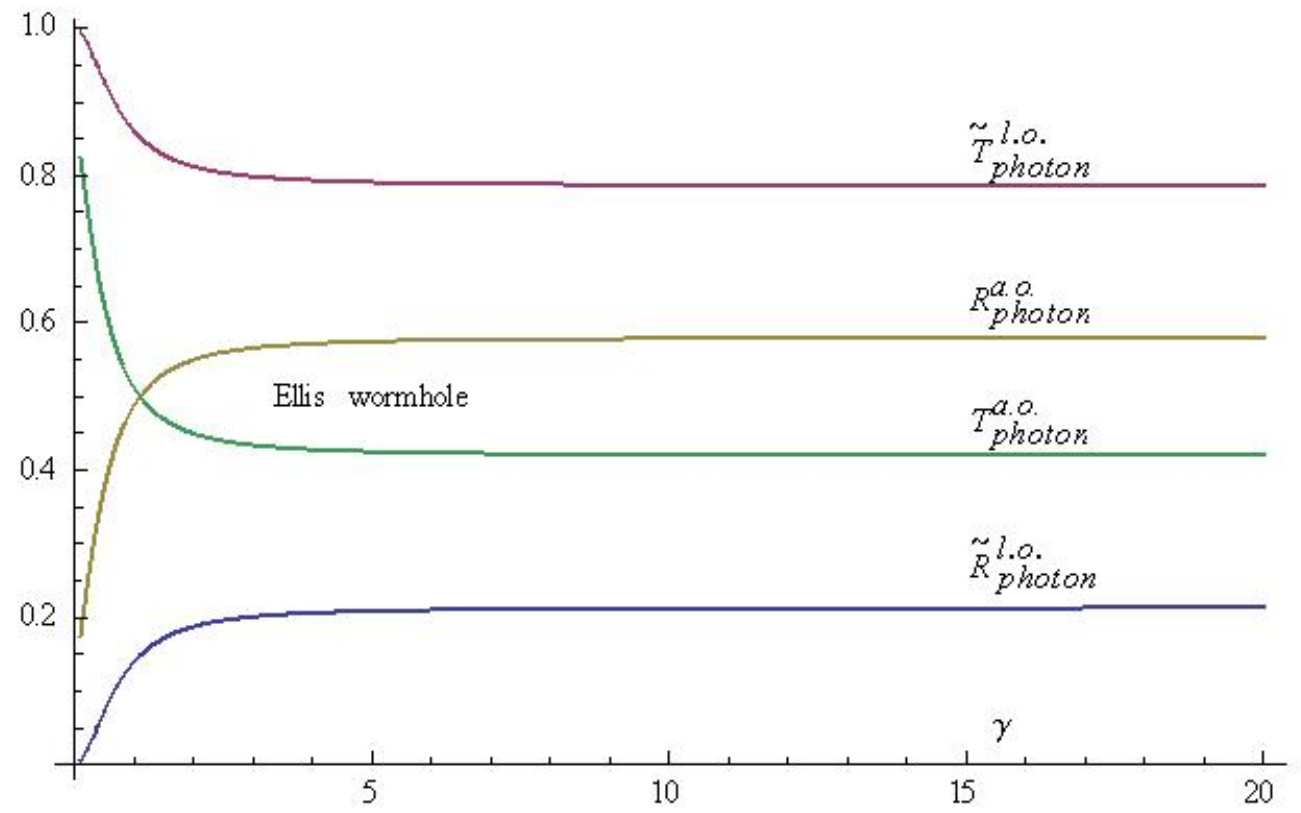

Figure 2: Reflection $(R)$ and Transmission $(T)$ probabilities for the ingoing light pulse in the Ellis wormhole spacetime. We choose units such that $M=1$ and plot $(R, T)$ at the throat, now only a function of $\gamma$ for different location of observers [Eqs.(64-67)]. Asymptotic observers are denoted by the superscript (a.o.) and local observers by (l.o.) with a tilde for coefficients. It turns out that $R$ and $T$ reach fixed values at some value of $\gamma$, hence at some fixed size of the throat $r_{\text {th }}$, where $R_{\text {photon }}^{\text {a.o. }} \simeq 0.60, T_{\text {photon }}^{\text {a.o. }} \simeq 0.40$, and $\widetilde{R}_{\text {photon }}^{\text {l.o. }} \simeq 0.20$ and $\widetilde{T}_{\text {photon }}^{\text {loo. }} \simeq 0.80$, as shown in the figure. The values are in contrast to the Schwarzschild values $R=1, T=0$ at the horizon, which suggests that the horizon plays the role of a perfect mirror totally reflecting away the incoming pulse. 


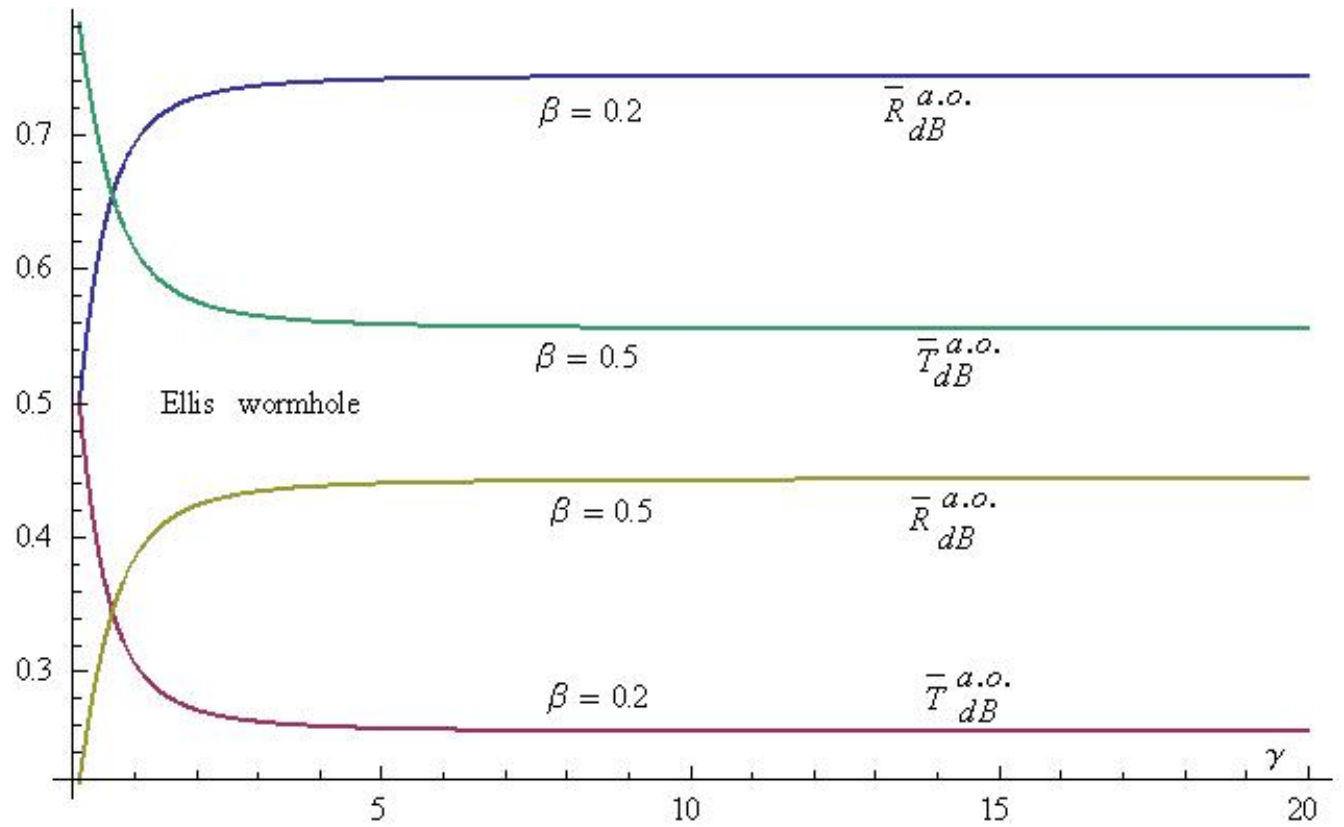

Figure 3: Reflection $(R)$ and Transmission $(T)$ probabilities for ingoing de Broglie waves in the Ellis wormhole spacetime as perceived by the asymptotic observers. We choose units such that $M=1$ and values $\alpha=\lambda^{\prime} / \lambda_{\mathrm{C}}=0.01$, $\beta=0.2$ and 0.5 . The plot $(R, T)$ at the throat, now only a function of $\gamma$ [Eqs. $(78,79)]$, shows that $R$ and $T$ reach fixed values at some value of $\gamma$, hence at some fixed size of the throat $r_{\text {th }}$. Asymptotic observers are denoted by the superscript (a.o.) for whom $\bar{R}_{\mathrm{dB}}^{\text {a.o. }} \sim 0.76, \bar{T}_{\mathrm{dB}}^{\text {a.o. }} \sim 0.24$, when $\beta=0.2$. Reflection probability is reduced to $\bar{R}_{\mathrm{dB}}^{\text {a.o. }}=0.45$, when $\beta$ is increased, e.g., to 0.5 , as shown in the figure. When $\beta \rightarrow 0$, stability is achieved: $\bar{R}_{\mathrm{dB}}^{\text {a.o. }} \rightarrow 1$, $\bar{T}_{\mathrm{dB}}^{\mathrm{a} . \mathrm{o}} \rightarrow 0$. 


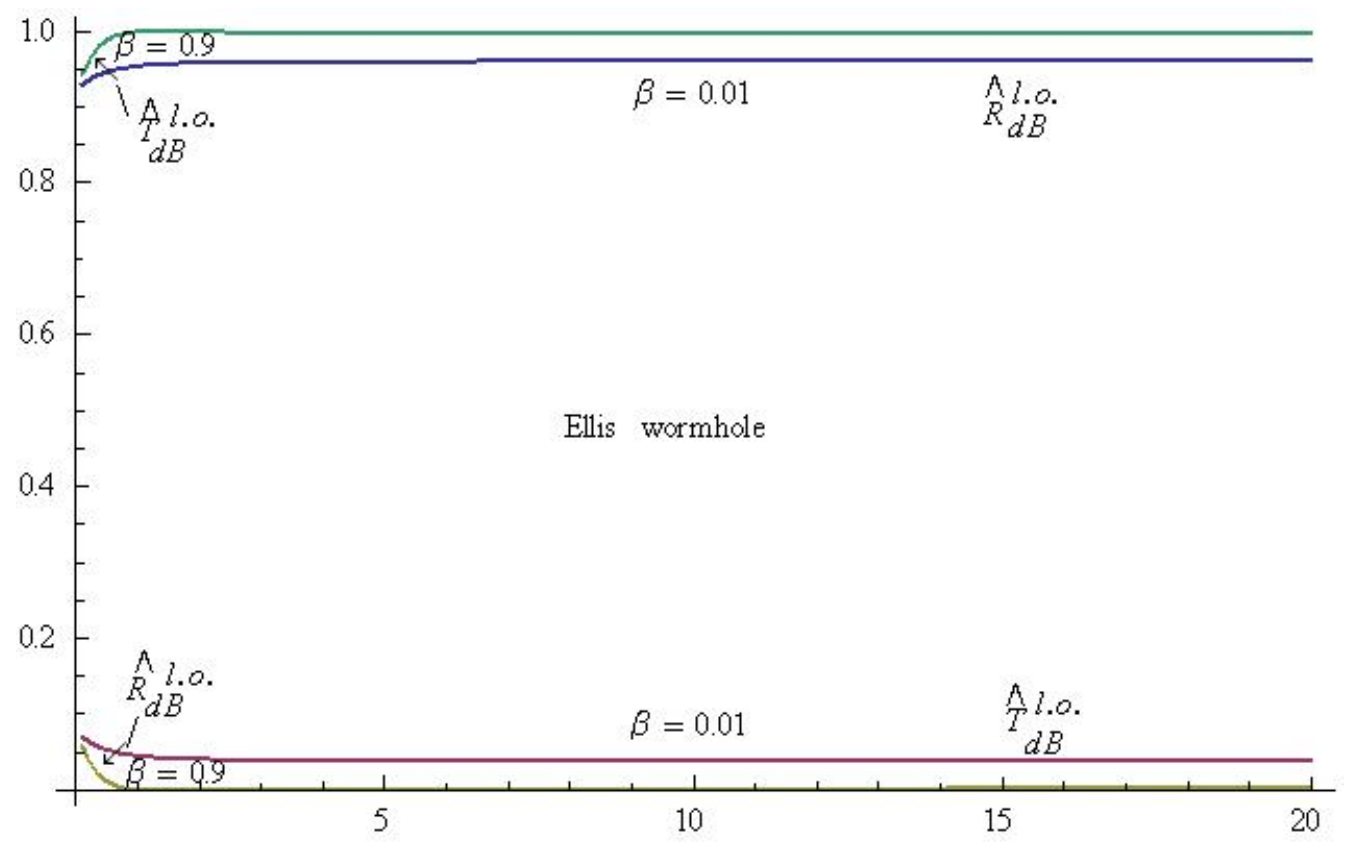

Figure 4: Reflection $(R)$ and Transmission $(T)$ probabilities for ingoing de Broglie waves in the Ellis wormhole spacetime perceived by the near-throat local observers. The plot $(R, T)$ at the throat, now only a function of $\gamma$ [Eqs. $(83,84)]$, shows de Broglie waves of high and low velocities $\beta=0.9$ and 0.01 . The lesser the velocity, the higher is the $\widehat{R}_{\mathrm{dB}}^{\text {l.o. }}$. Stability is achieved: $\widehat{R}_{\mathrm{dB}}^{\text {l.o. }} \rightarrow 1, \widehat{T}_{\mathrm{dB}}^{\text {l.o. }} \rightarrow 0$ only in the limit $\beta \rightarrow 0$. 


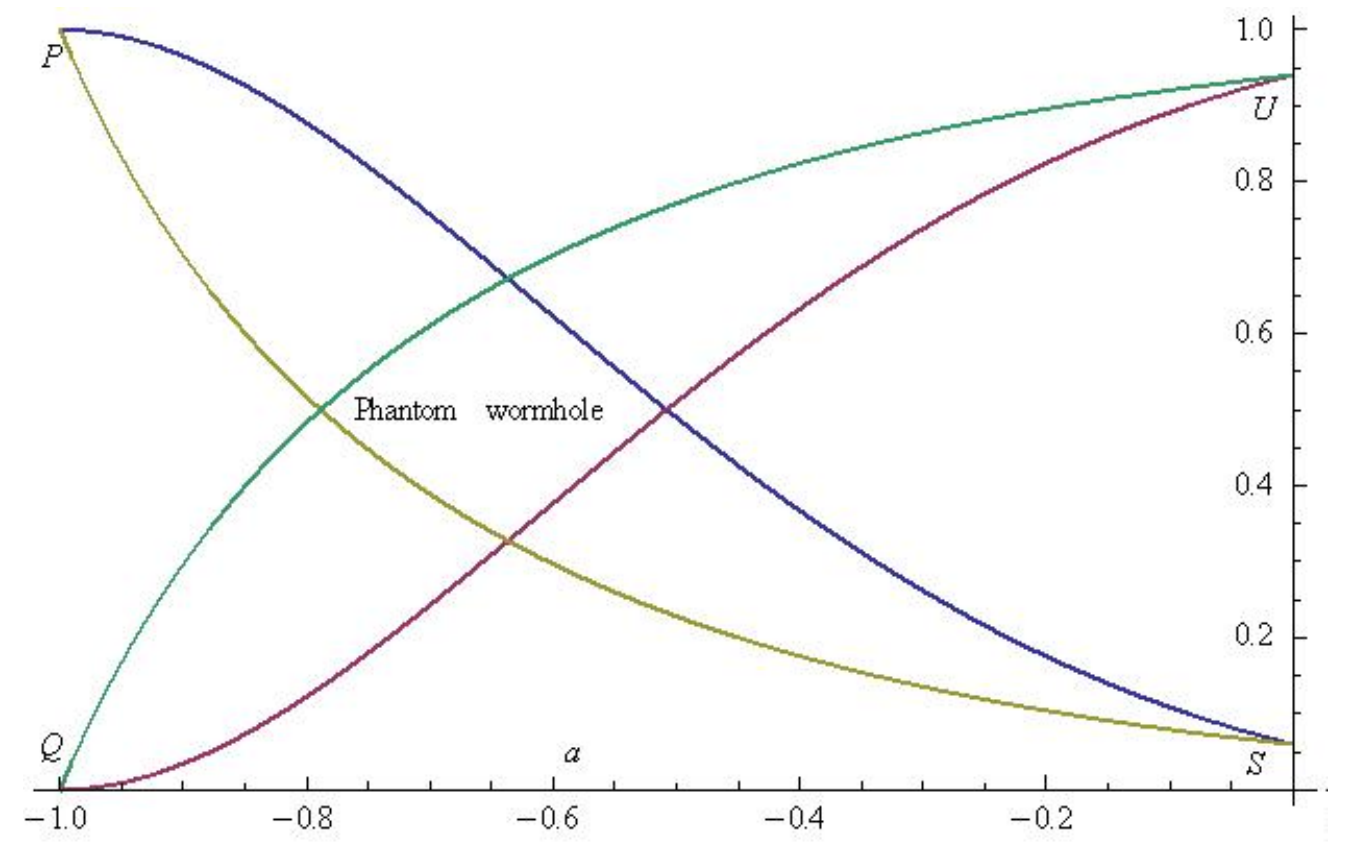

Figure 5: Reflection $(R)$ and Transmission $(T)$ probabilities for de Broglie waves in the phantom wormhole spacetime $(-1<a<0)$. The point $P$ corresponds to $R_{\text {photon }}^{\text {a.o. }}=\widetilde{R}_{\text {photon }}^{\text {l.o. }}=1, \bar{R}_{\mathrm{dB}}^{\text {a.o. }}=\widehat{R}_{\mathrm{dB}}^{\text {l.o. }}=1$ and $Q$ corresponds to $T_{\text {photon }}^{\text {a.o. }}=\widetilde{T}_{\text {photon }}^{\text {l.o. }}=0, \bar{T}_{\mathrm{dB}}^{\text {a.o. }}=\widehat{T}_{\mathrm{dB}}^{\text {l.o. }}=0$. See Eqs. $(105,106)$. Likewise, $S$ corresponds to $R_{\text {photon }}^{\text {a.o. }}=\widetilde{R}_{\text {photon }}^{\text {l.o. }}=0.06, \bar{R}_{\mathrm{dB}}^{\text {a.o. }}=\widehat{R}_{\mathrm{dB}}^{\text {l.o. }}=0.06$ and $U$ corresponds to $T_{\text {photon }}^{\text {a.o. }}=\widetilde{T}_{\text {photon }}^{\text {l.o. }}=0.94, \bar{T}_{\mathrm{dB}}^{\text {a.o. }}=\widehat{T}_{\mathrm{dB}}^{\text {l.o. }}=0.94$. See Eqs. $(107,108)$. 